\title{
On the abundance of gravitational arcs produced by submillimeter galaxies at radio and submm wavelengths
}

\author{
C. Fedeli ${ }^{1,2,3}$ and A. Berciano Alba ${ }^{4,5}$ \\ 1 Dipartimento di Astronomia, Università di Bologna, via Ranzani 1, 40127 Bologna, Italy \\ e-mail: cosimo. fedeli@unibo.it \\ 2 INAF - Osservatorio Astronomico di Bologna, via Ranzani 1, 40127 Bologna, Italy \\ 3 INFN, Sezione di Bologna, Viale Berti Pichat 6/2, 40127 Bologna, Italy \\ ${ }^{4}$ Netherlands Institute for Radio Astronomy, Postbus 2, 7990 AA, Dwingeloo, The Netherlands \\ e-mail: berciano@astro.rug.nl \\ 5 Kapteyn Astronomical Institute, University of Groningen, PO Box 800, 9700 AV, Groningen, The Netherlands
}

Received 14 July 2009 / Accepted 7 September 2009

ABSTRACT

\begin{abstract}
We predict the abundance of giant gravitational arcs produced by submillimeter galaxies (SMGs) lensed by foreground galaxy clusters, both at radio and submm wavelengths. The galaxy cluster population is modeled in a realistic way with the use of semi-analytic merger trees, while the density profiles of individual deflectors take into account ellipticity and substructures. The adopted typical size of the radio and submm emitting regions of SMGs is based on current radio/CO observations and the FIR-radio correlation. The source redshift distribution has been modeled using three different functions (based on spectroscopic/photometric redshift measurements and a simple evolutionary model) to quantify the effect of a high redshift tail on the number of arcs. The source number counts are compatible with currently available observations, and were suitably distorted to take into account the lensing magnification bias. We present tables and plots for the numbers of radio and submm arcs produced by SMGs as a function of surface brightness, useful for the planning of future surveys aimed at arc statistics studies. They show that e.g., the detection of several hundred submm arcs on the whole sky with a signal-to-noise ratio of at least 5 requires a sensitivity of $1 \mathrm{mJy} \operatorname{arcsec}^{-2}$ at $850 \mu \mathrm{m}$. Approximately the same number of radio arcs should be detected with the same signal-to-noise ratio with a surface brightness threshold of $20 \mu \mathrm{Jy} \operatorname{arcsec}^{-2}$ at $1.4 \mathrm{GHz}$. Comparisons of these results with previous work found in the literature are also discussed.
\end{abstract}

Key words. gravitational lensing - galaxies: clusters: general - radio continuum: galaxies - submillimeter

\section{Introduction}

The effect of gravitational lensing constitutes a unique research tool in many astrophysical fields, since it allows one to investigate the structures of both the lenses (e.g., galaxies and galaxy clusters) and the lensed background sources, as well as to probe the three-dimensional mass distribution of the Universe. In particular, one of the most spectacular phenomena associated with the gravitational deflection of light are the giant arcs observed in galaxy clusters, which are caused by extended background sources lying in the regions where the lensing magnification produced by the cluster is strongest. The effective source magnification can easily reach $\sim 30$ in these cases, providing the opportunity to detect and spatially resolve the morphologies and internal dynamics of high redshift background sources at a level of detail far greater than otherwise possible. An illustration of this powerful technique was presented in Swinbank et al. (2007), where a magnification factor of 16 by the cluster RCS 0224-002 allowed them to study the star formation activity, mass and feedback processes of a Lyman break galaxy at $z \sim 5$, something that (without the help of lensing) would not be possible beyond $z \sim 2$ with current instruments.

A particularly interesting application of gravitational lensing is the so-called arc statistics, i.e. the study of the abundance of large tangential arcs in galaxy clusters. Among other things, this abundance is sensitive to the cluster mass function, the cluster dynamical activity (e.g., infall of matter, mergers, etc.) and internal structure of host dark-matter halos (e.g., triaxiality and the concentration of the density profile), which makes arc statistics a unique tool to study the cluster population. Arc statistics studies at optical and near-infrared wavelengths have been numerous in the past decade on both the observational (Luppino et al. 1999; Gladders et al. 2003; Zaritsky \& Gonzalez 2003) and theoretical sides (Bartelmann \& Weiss 1994; Wambsganss et al. 1998; Bartelmann et al. 2003; Meneghetti et al. 2003; Wambsganss et al. 2005; Fedeli et al. 2008).

All the currently known giant arcs come from detections in the optical. However, the fraction of lensed sources observed in the $\mathrm{mm} / \mathrm{submm}$ wavebands is expected to be much larger than in the optical (Blain 1996, 1997): Due to the spectral shape of the thermal dust emission, the observed submm flux density of dusty galaxies with a given luminosity remains approximately constant in the redshift range $1 \lessgtr z \lessgtr 8$ instead of declining with increasing distance (usually referred to as "negative $K$-correction", see Blain \& Longair 1993, 1996; Blain et al. 2002). This effect, together with the steep slope of the observed submm number counts, produces a strong magnification bias that makes submm galaxies (hereafter SMGs) an ideal source population for the production of lensed arcs.

The SMGs were first detected about a decade ago with SCUBA $^{1}$ (Smail et al. 1997; Hughes et al. 1998; Barger et al. 1998; Eales et al. 1999). The current observational evidence

\footnotetext{
1 Submillimeter Common User Bolometer Array (Holland et al. 1999), which used to be mounted at the James Clerk Maxwell Telescope (JCMT) located in Hawaii.
} 
indicates that these objects are high-redshift dust obscured galaxies, in which the rest frame FIR peak of emission is observed in the submm band. Their FIR luminosities, in the range $10^{11}-10^{13} L_{\odot}$, are $\sim 100$ times higher than what is observed in local spirals. Their energy output seems to be dominated by star formation processes induced by galaxy interactions/mergers, although a good fraction ( $30-50 \%)$ of SMGs also seems to host an Active Galactic Nucleus (AGN, e.g. Alexander et al. 2005; Michałowski et al. 2009). The available evidence also suggests that SMGs might be the progenitors of massive local ellipticals (Lilly et al. 1999; Smail et al. 2002, 2004; Webb et al. 2003; Genzel et al. 2003; Alexander et al. 2003, 2005; Swinbank et al. 2006, 2008; Michałowski et al. 2009).

In the $\sim 30$ clusters observed with SCUBA (Smail et al. 2002; Chapman et al. 2002; Cowie et al. 2002; Knudsen et al. 2008), only 4 multiply imaged SMGs have been reported to date (Borys et al. 2004; Kneib et al. 2004; Knudsen et al. 2008). This extremely low detection rate is due to three major limitations of current $850 \mu$ m surveys: (i) very small sky coverage ( $\sim 3$ square degrees, including all cluster and blank field surveys); (ii) confusion limited maps at $\sim 2 \mathrm{mJy}$ (which means that only the brightest members are detected) and (iii) insufficient resolution $\left(\sim 15^{\prime \prime}\right)$ to resolve extended lensed structures. Current efforts to increase the surveyed area at $850 \mu \mathrm{m}$ include the $\mathrm{SASS}^{2}$, the $\mathrm{SCLS}^{3}$ and the all sky survey that will be carried out with the HFI bolometer on board of the Planck satellite ${ }^{4}$, but their resolutions will still be insufficient to identify lensed arcs. The only instrument that can currently provide sub-arcsecond resolution in submm (at $890 \mu \mathrm{m}$ ) is the $\mathrm{SMA}^{5}$.

However, the tight correlation between radio synchrotron and FIR emission observed in star-forming galaxies (van der Kruit 1973; Helou et al. 1985), provides an alternative way to obtain high resolution images of SMGs. Commonly referred to as "the FIR/radio correlation", it covers about five orders of magnitude in luminosity (Condon 1992; Garrett 2002) out to $z \sim 3$ (Kovács et al. 2006; Vlahakis et al. 2007; Ibar et al. 2008; Michałowski et al. 2009). The standard model about its nature considers that both emissions are caused by massive star formation: while young massive stars produce UV radiation that is re-emitted in the FIR by the surrounding dust, old massive stars explode as supernovae, producing electrons that are accelerated by the galactic magnetic field and generate the observed radio synchrotron emission (Harwit \& Pacini 1975; Helou et al. 1985). Therefore, given the possible common physical origin of both emissions, radio interferometric observations can be used as a high-resolution proxy for the rest-frame FIR emission of high- $z$ galaxies observed in the submm.

The advent of ALMA $^{6}$ will open a new window into $\mathrm{mm} /$ submm astronomy at sub-arcsecond resolution and sub-mJy sensitivity, allowing the detection of resolved gravitational arcs produced by SMGs. Although its small instantaneous field of view (FOV) severely limits ALMA's survey capability, a $25 \mathrm{~m}$ submm telescope $\left(\mathrm{CCAT}^{7}\right)$ is going to be built on a high peak in the Atacama region to provide wide field images $\left(\sim 400 \mathrm{arcmin}^{2}\right)$ with a resolution of $\sim 3.5^{\prime \prime}$ at $350 \mu \mathrm{m}$. With the combined

2 SCUBA-2 All Sky Survey, a $\sim 2 \times 10^{4}$ square degree survey with a $5 \sigma$ depth of $150 \mathrm{mJy}$ and $15^{\prime \prime}$ resolution.

3 SCUBA-2 Cosmology Legacy Survey, a $\sim 35$ square degree survey with a $5 \sigma$ depth of $3.5 \mathrm{mJy}$ and $15^{\prime \prime}$ resolution.

$45 \sigma$ depth of $\sim 350 \mathrm{mJy}$ and $\sim 50^{\prime \prime}$ resolution.

5 The Submillimeter Array in Hawaii.

6 The Atacama Large Millimeter Array in Chile.

7 Cornell Caltech Atacama Telescope. capabilities of both instruments, arc statistics studies in the submm might be possible.

At the same time, radio interferometry is also experiencing major technological improvements. In particular, the VLA ${ }^{8}$ and MERLIN $^{9}$ are currently undergoing major upgrades which will boost their sensitivities by factors of 10-30 and dramatically improve their mapping capabilities. The new versions of these arrays (e-MERLIN and EVLA) will be fully operational in 2010 and 2012 respectively. In order to assess the prospects for the study of gravitationally lensed arcs at submm and radio wavelengths, in this work we report detailed theoretical predictions about the abundance of arcs produced by the SMG population at $850 \mu \mathrm{m}$ and $1.4 \mathrm{GHz}$.

The paper is organized as follows. In Sect. 2, we introduce all the relevant quantities that are necessary to derive the total number of arcs detectable on the sky. In Sect. 3 we present and discuss all the observational information about SMGs that is required for the subsequent strong lensing analysis: morphology, redshift distribution and number counts. A description of our cluster population model and the way in which the abundance of large arcs is computed is given in Sect. 4. The derived arc redshift distributions and number of arcs are presented in Sect. 5, while in Sect. 6 we discuss how these relate to previous findings in the literature. A summary and conclusions are presented in Sect. 7.

The adopted cosmology corresponds to the standard $\Lambda \mathrm{CDM}$ model with cosmological parameters inferred from the WMAP-5 data release in conjunction with type Ia supernovae and baryon acoustic oscillation datasets (Dunkley et al. 2009; Komatsu et al. 2009), namely $\Omega_{\mathrm{m}, 0}=0.279, \Omega_{\Lambda, 0}=0.721$, $\sigma_{8}=0.817$ and $H_{0}=h 100 \mathrm{~km} \mathrm{~s}^{-1} \mathrm{Mpc}^{-1}$, with $h=0.701$.

\section{Strong lensing statistics}

The choice of the best parameters to be used in order to characterize the morphological properties of long and thin gravitational arcs is still a matter of debate. In this work, we adopted the quite popular choice of the length-to-width radio $d$, which has to be larger than a certain threshold $d_{0}$ (usually 7.5 or 10) in order to consider an object as a giant arc.

Given a set of background sources placed at redshift $z_{\mathrm{s}}$, the efficiency of the galaxy cluster population to produce arcs with length-to-width ratio $d \geq d_{0}$, is parametrized by the optical depth

$\tau_{d_{0}}\left(z_{\mathrm{s}}\right)=\frac{1}{4 \pi D_{\mathrm{s}}^{2}} \int_{0}^{z_{\mathrm{s}}} \int_{0}^{+\infty} n(M, z) \sigma_{d_{0}}(M, z) \mathrm{d} M \mathrm{~d} z$

where $D_{\mathrm{s}}$ is the angular diameter distance to the source redshift and $n(M, z)$ is the total number of clusters present in the unit redshift around $z$ with mass in the unit interval around $M$. The cross section $\sigma_{d_{0}}(M, z)$ is the area of the region on the source plane where a source has to lie in order to produce (at least) one gravitational arc with $d \geq d_{0}$, for a single cluster with mass $M$ at redshift $z$. This depends in general on the cluster structure, the source properties, and the redshifts of both the cluster and the source. Since in realistic situations sources are distributed at different redshifts, we can calculate the average optical depth by integrating $\tau_{d_{0}}\left(z_{\mathrm{s}}\right)$ over the source redshift distribution $p\left(z_{\mathrm{s}}\right)$,

$\bar{\tau}_{d_{0}}\left(z_{\mathrm{s}}\right)=\int_{0}^{z_{\mathrm{s}}} p(\xi) \tau_{d_{0}}(\xi) \mathrm{d} \xi$

\footnotetext{
8 The Very Large Array in New Mexico.

9 The UK Multi-Element Radio Linked Interferometer Network.
} 
In this way, the total number of arcs with length-to-width ratio $d \geq d_{0}$ can be calculated as (Bartelmann et al. 1998)

$\mathcal{N}_{d_{0}}=4 \pi N \bar{\tau}_{d_{0}}$

where $N$ is the observed surface density of sources, and $\bar{\tau}_{d_{0}}=$ $\bar{\tau}_{d_{0}}(+\infty)$ is the total average optical depth (i.e., the average optical depth with the integral extending to all possible source redshifts). Therefore, the number of arcs produced by a given population of background sources can be calculated by providing the following observational constraints: (i) the characteristic source shape and size, which is necessary to compute the cluster cross sections $\sigma_{d_{0}}(M, z)$; (ii) the source redshift distribution $p\left(z_{s}\right)$, which is necessary to evaluate the optical depth $\bar{\tau}_{d_{0}}$; and (iii) the cumulative source number counts $N$.

\section{Characteristics of the SMG population}

\subsection{Source shape and size}

In strong lensing statistics studies, it is customary to characterize the size of elliptical background sources using the equivalent effective radius $R_{\mathrm{e}} \equiv \sqrt{a b}$, which is the radius of a circle that has the same area of the elliptical source with semi-major axis $a$ and semi-minor axis $b$. In addition, the orientation of sources is randomly chosen, and to account for the different source shapes the value of the axis ratio $b / a$ is considered to vary within a certain interval. The typical values of these parameters used in optical ray-tracing simulations are $R_{\mathrm{e}}=0.5^{\prime \prime}$ and $b / a$ randomly varying in the interval $[0.5,1]$ (Meneghetti et al. 2000, 2003, 2005; Fedeli et al. 2006).

Due to the low resolution of submm single-dish observations (e.g., $F W H M \sim 15^{\prime \prime}$ for SCUBA at $850 \mu \mathrm{m}$ ), current estimates of the typical size of SMGs are based on continuum radio (Chapman et al. 2004; Biggs \& Ivison 2008) and millimeter (e.g. Tacconi et al. 2006) interferometric observations of small source samples. In particular, Biggs \& Ivison (BI08 hereafter) combined $1.4 \mathrm{GHz}$ data from the VLA and MERLIN to produce high resolution radio maps of $12 \mathrm{SMGs}$ detected in the Lockman Hole. The deconvolved sizes derived from these radio maps (obtained by fitting each map with an elliptical Gaussian) are consistent with Chapman et al. (2004) (see also Muxlow et al. 2005) and Tacconi et al. (2006).

Figure 1 shows the effective radius and axis ratio for each of the 12 radio sources reported in BI08 (black circles). Note that, although the $b / a$ interval $[0.5,1]$ used in optical lensing simulations contains 11 out of the 12 radio sources, there are several error bars that extend below its lower limit. In addition, the optical effective radius of $0.5^{\prime \prime}$ is not suitable to describe them. Since the source sample is too small (and the error bars rather large) to derive a reliable size distribution, we decided to use an effective radius close to the median of the sample. As a result, the size of the $1.4 \mathrm{GHz}$ radio emission produced by the SMG population has been characterized in the following by $b / a$ randomly varying within $[0.3,1]$ and $R_{\mathrm{e}}=0.25^{\prime \prime}$.

Since SMGs seem to follow the FIR-radio correlation (e.g. Kovács et al. 2006), their emission at both $1.4 \mathrm{GHz}$ and $850 \mu \mathrm{m}$ is expected to be associated with massive star formation. This means that, as a first approximation, the same morphological parameters can be used to characterize the sizes and shapes of SMGs at radio and submm wavelengths. This choice of parameters for the submm emission is also consistent with the recent SMA observations presented by Younger et al. (2008), which have partially resolved the $890 \mu \mathrm{m}$ emission of a SMG (GN20) for the first time (see Fig. 1).

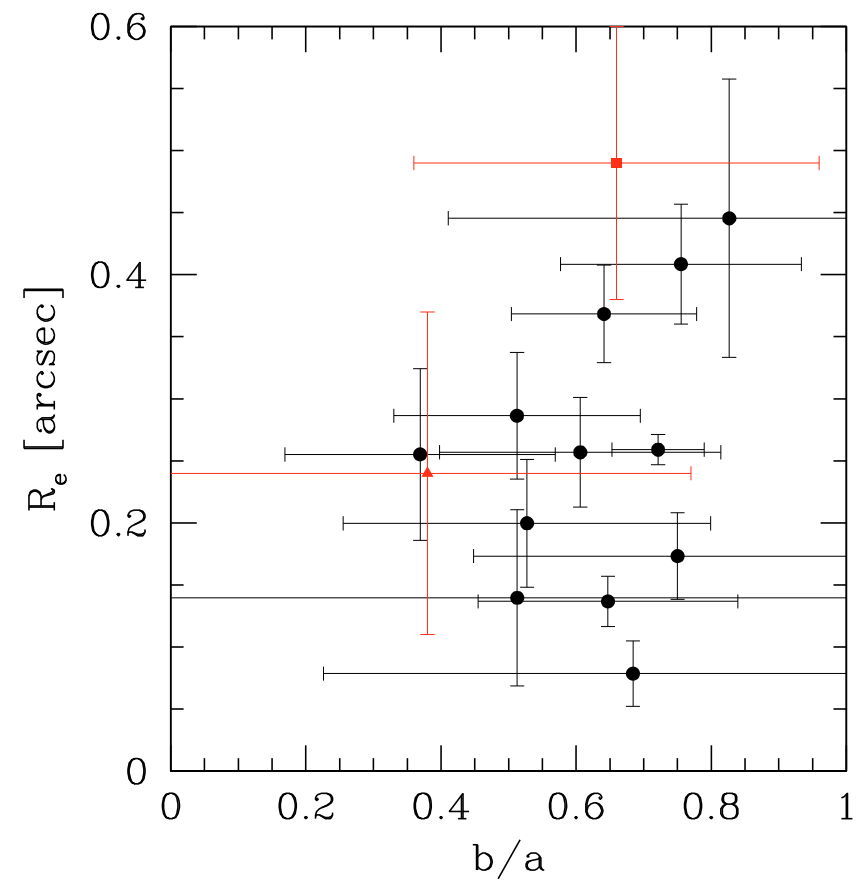

Fig. 1. Effective radii and axis ratios derived for the $1.4 \mathrm{GHz}$ radio counterparts of the 12 SMGs presented in Biggs \& Ivison (2008). The red points correspond to the $890 \mu \mathrm{m}$ counterpart of GN20 detected with the SMA, whose size was derived by fitting a Gaussian (triangle) and an elliptical disk (square) to the data (Younger et al. 2008). Error bars are computed through standard error propagation.

If we wish to characterize gravitational arcs via their lengthto-width ratio and make comparisons between observations and theoretical predictions in an unbiased way, it is crucial to resolve their width. To address if the resolution provided by radio and submm instruments could be an issue for arc statistics studies, we investigated the width distribution of arcs produced by a population of sources that is being lensed by a galaxy cluster. In particular, we used the most massive lensing cluster at $z=0.3$ from the MareNostrum cosmological simulation (Gottlöber \& Yepes 2007), a large $n$-body and gas-dynamical run, whose lensing properties recently have been studied by Fedeli et al. (2009, in preparation). The mass distribution of this cluster was projected along three orthogonal directions, for which we derived deflection angle maps by standard ray-tracing techniques (Bartelmann \& Weiss 1994). Then, a set of sources at $z_{\mathrm{s}}=1$ (source redshift at which the lensing efficiency peaks for lenses at $z \sim 0.3$ ) with $R_{\mathrm{e}}=0.25^{\prime \prime}$ and axis ratios randomly varying in the interval $[0.3,1]$ was lensed through the three projections. As usual in this procedure, the sources are preferentially placed near the lensing caustics following an iterative procedure to enhance the probability of the production of large arcs. The bias introduced by this artificial increase of sources is corrected for by assigning a weight $\leq 1$ to each source, which is reduced at each new iteration step (see Miralda-Escude 1993; Bartelmann \& Weiss 1994; Bartelmann et al. 1998, for further details).

The black histogram shown in Fig. 2 corresponds to the width distribution of arcs derived from this simulation. For comparison, we have also included the corresponding distribution derived from the parameters used in optical lensing simulations (blue histogram). The two panels illustrate the difference between selecting arcs characterized by $d \geq d_{0}$ with $d_{0}=7.5$ (left) and $d_{0}=10$ (right). As expected, reducing the source equivalent size produces a decrease in the width of lensed images. 

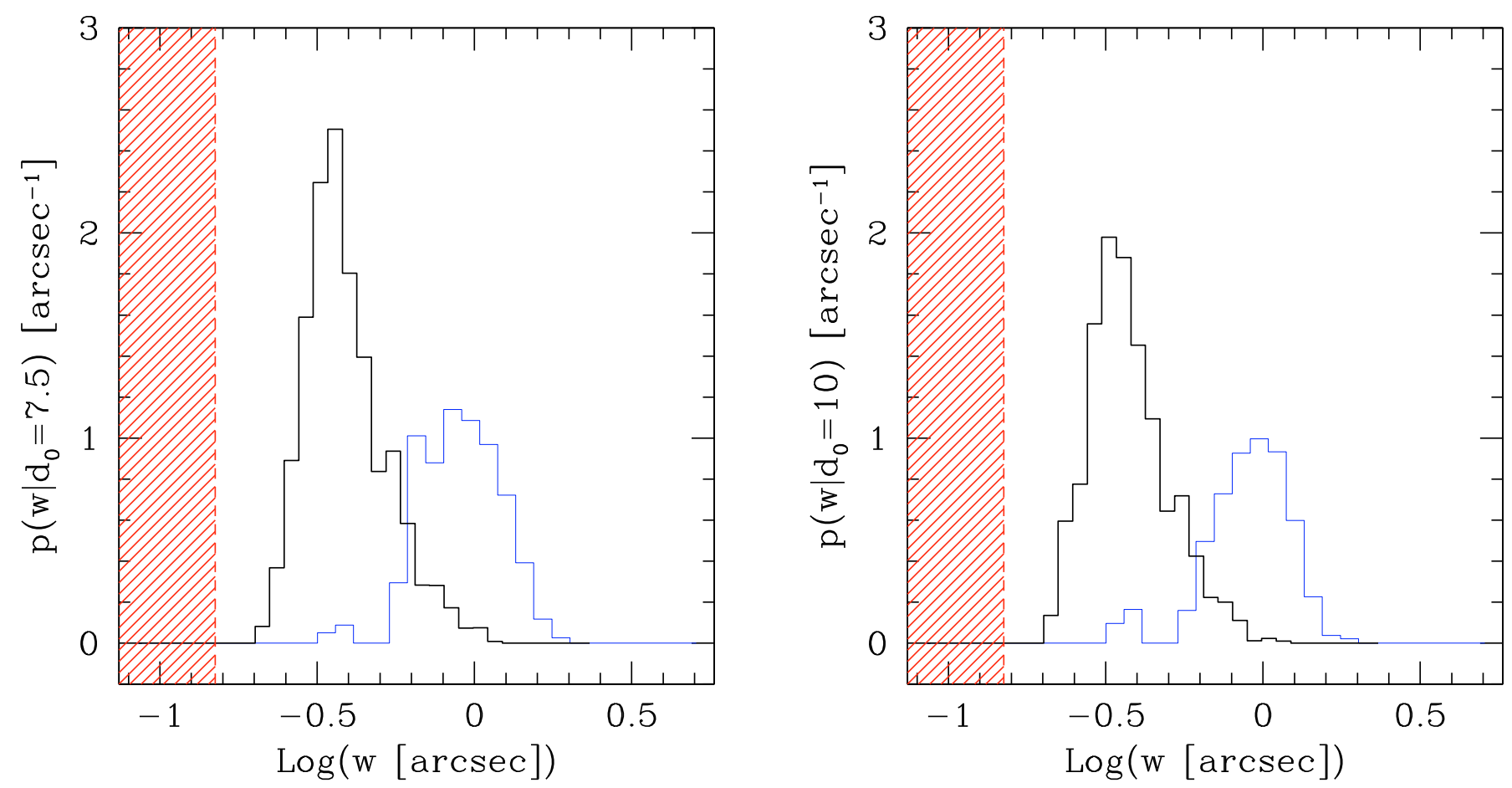

Fig. 2. Arc width probability distributions derived from a set of sources at $z_{\mathrm{s}}=1$ that were lensed by (the three projections of) the most massive cluster of the MareNostrum simulation at $z=0.3$. The two panels illustrate the difference between selecting arcs with a length-to-width ratio $d \geq d_{0}=7.5$ (left) and $d \geq d_{0}=10$ (right). The black histograms were produced using the typical source size of SMGs at $1.4 \mathrm{GHz}$ and $850 \mu \mathrm{m}$ assumed in this work $\left(R_{\mathrm{e}}=0.25^{\prime \prime}\right.$ and $b / a$ randomly varying in the interval $\left.[0.3,1]\right)$. The blue histograms correspond to optical sources as usually described in lensing simulations $\left(R_{\mathrm{e}}=0.5^{\prime \prime}\right.$ and $\left.b / a \in[0.5,1]\right)$. The red dashed region shows the 150 mas resolution limit of the $e$-MERLIN radio interferometer at $1.4 \mathrm{GHz}$.

Note also that the behavior of the distributions is practically independent of the minimum length-to-width ratio used to select the arcs. The most important feature, however, is that both distributions drop to zero for widths below $\sim 0.2^{\prime \prime}$, meaning that virtually no radio/submm (or optical) arcs have widths smaller than that value. At $1.4 \mathrm{GHz}, \sim 0.2^{\prime \prime}$ resolution is already accessible with MERLIN/e-MERLIN $\left(\sim 0.15^{\prime \prime}\right)$. Therefore, resolving the width of long and thin images for arc statistics studies is in principle already possible at radio wavelengths. However, until the advent of ALMA, the $\sim 0.75^{\prime \prime}$ resolution of the SMA at $950 \mu \mathrm{m}$ will only be able to resolve a very small fraction of arcs produced by the most extended SMGs.

Finally, we would like to stress two points related to the choice of source morphological parameters presented in this section. First, the most luminous SMGs seem to be the result of merger processes (e.g., Greve et al. 2005; Tacconi et al. 2006), hence it is unlikely that their true shape is elliptical, as we assumed. However, if a merging source is lensed as an arc at a particular wavelength, irregularities in its shape and internal structure will not significantly change the global morphological properties of the arc, like the length-to-width ratio. What can happen is that the length-to-width ratio of an arc changes with wavelength because the emitting region of the source at those wavelengths have different sizes. For some of the wavelengths the source might even look like a group of small isolated emitting regions instead of a continuous one, which means that in the image plane it will be observed as a group of disconnected multiple images instead of a full arc. A very illustrative example of this scenario is SMM J04542-0301, an elongated region of submm emission which seems to be associated with a merger at $z=2.9$ that is being lensed by the cluster MS0451.6-0305
(Borys et al. 2003; Berciano Alba et al. 2007; Berciano Alba et al. 2009). Until more complete information about the average structure of SMGs becomes available, we believe our approach to be the best that can be done.

Second, since the radio sources studied by BI08 are brighter than $50 \mu \mathrm{Jy}$, the typical size derived from this sample might be different from the one that could be derived from fainter SMGs. Note, however, that the change of the cluster cross section with source size has a very small slope for $R_{\mathrm{e}}$ between $0.2^{\prime \prime}$ and $1.5^{\prime \prime}$ (Fedeli et al. 2006). Therefore, deviations from $R_{\mathrm{e}}=0.25^{\prime \prime}$ within this interval (which is two times larger than the interval that contains the sizes measured by BI08 and Tacconi et al. (2006), see Fig. 6 of BI08) will have a negligible effect on the derived number of arcs.

\subsection{Source redshift distribution}

A key point in trying to estimate the abundance of strong lensing features that are produced by the galaxy cluster population is the redshift distribution of background sources. Distributions peaked at higher redshift, or with a substantial high- $z$ tail, will have in general more potential lenses at their disposal, and hence will produce larger arc abundances as compared to low$z$-dominated distributions. In addition, the lensing efficiency for individual deflectors is also an increasing function of the source redshift.

The most robust estimate of the redshift distribution of SMGs to date is based on the $\sim 15 \operatorname{arcmin}^{2}$ SCUBA survey carried out by Chapman et al. (2005) (CH05 hereafter). Radio observations were used to pinpoint the precise location of the submm detections, allowing the identification of optical counterparts that could provide precise spectroscopic redshifts. 

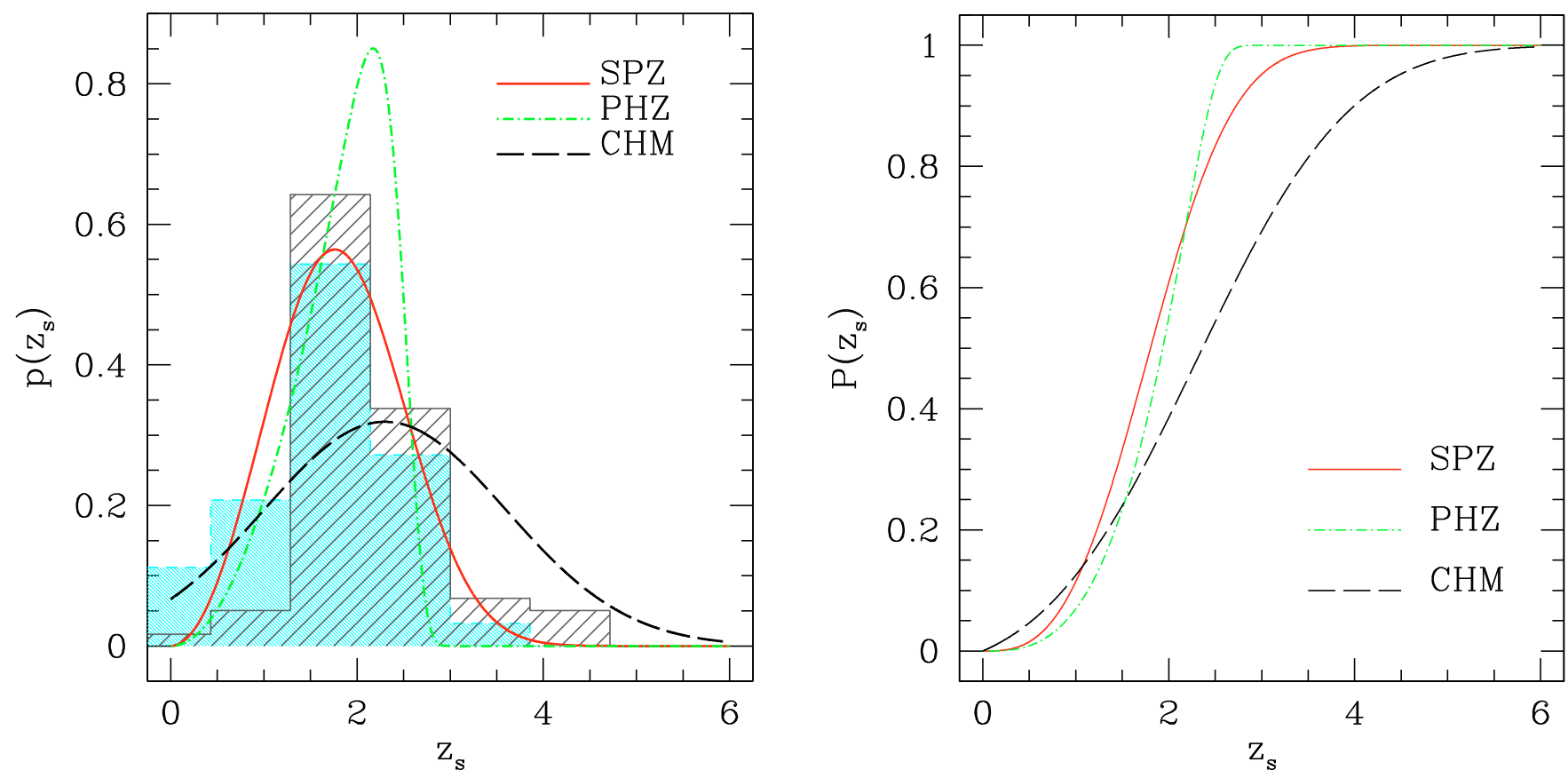

Fig. 3. Left panel. Redshift distribution of SMGs derived from the spectroscopic sample of Chapman et al. (2005) (cyan histogram) and the photometric sample of Aretxaga et al. (2007) (dark-grey histogram). The curves SPZ and PHZ correspond to the best fits provided by Eq. (4) to the spectroscopic and photometric data, respectively. The curve CHM corresponds to the best Gaussian fit to the simple evolutionary model for SMGs used in $\mathrm{CHO} 5$ (as quoted in $\mathrm{CH} 05$ ), normalized to the redshift interval $[0,+\infty]$. Note that the binning adopted here is different from the one used in $\mathrm{CH} 05$, so the "redshift desert" in the redshift interval $z=1.2-1.8$ is not evident. However, changing the binning of the histogram changes little of the subsequent fit. Right panel. The cumulative distributions corresponding to the fits reported on the left panel, calculated by using Eq. (5).

The final sample is composed of 73 SMGs with $850 \mu \mathrm{m}$ flux densities $>3 \mathrm{mJy}$ and radio counterparts with flux densities at $1.4 \mathrm{GHz}>30 \mu \mathrm{Jy}$.

On the other hand, the SCUBA Half-Degree Extragalactic Survey (SHADES, Mortier et al. 2005; van Kampen et al. 2005) is the largest $\left(720 \operatorname{arcmin}^{2}\right) 850 \mu \mathrm{m}$ survey to date ${ }^{10}$. From their catalog of 120 SMGs, 69 have robust radio counterparts with $S_{850 \mu \mathrm{m}} \geq 3 \mathrm{mJy}$ and $S_{1.4 \mathrm{GHz}} \geq 20 \mu \mathrm{Jy}$. Photometric redshifts for this sub-sample were calculated by Aretxaga et al. (2007) (AR07 hereafter) by fitting Spectral Energy Distribution (SED) templates to the available photometry at $850 \mu \mathrm{m}$ and $1.4 \mathrm{GHz}$, including upper limits at $450 \mu \mathrm{m}$ (additional photometry at millimeter wavelengths was also used for 13 out of the 69 sources). The histogram of the resulting photometric redshift distribution, together with the spectroscopic one reported by $\mathrm{CH} 05$, are shown in Fig. 3. The accuracy on the photometric redshifts derived by $A R 07$ is $\Delta z \sim 0.65$. Note that the requirement for a radio counterpart biases these two redshift distributions against SMGs with $z>3$, due to the less favorable $K$-correction in the radio compared with submm. Using a simple evolutionary model, CH05 estimated that the fraction of SMGs $\left(S_{850 \mu \mathrm{m}}>5 \mathrm{mJy}\right)$ that is missing between $z \sim 2.5$ and $z \sim 5$ in their spectroscopic redshift distribution is $\sim 35 \%$, a number that is in agreement with the fraction of radio-unidentified SMGs reported in Ivison et al. (2002); Chapman et al. (2003) and the SHADES survey (AR07). The model evolves the local FIR luminosity function in luminosity with increasing redshift following the prescription of Blain et al. (2002). To account for the dust properties of SMGs, it also includes a range of SED templates that have been tuned to fit

10 The largest SMG survey to date $\left(0.7 \mathrm{deg}^{2}\right)$ has being carried out at $1.1 \mathrm{~mm}$ with the AzTEC continuum camera mounted on the JCMT (Austermann et al. 2009). their observed submm flux distribution (Chapman et al. 2003; Lewis et al. 2005).

In order to investigate the effect of this high- $z$ tail on the predicted number of arcs, we used three different analytic expressions to characterize the redshift distribution of SMGs in our calculations (see Fig. 3). One of them (CHM) corresponds to the best Gaussian fit to the distribution predicted by the $\mathrm{CH} 05$ evolutionary model, as quoted in CH05. The other two (SPZ and PHZ) were obtained by fitting the $\mathrm{CH} 05$ and AR07 histograms with the following analytic function, usually adopted for optical strong lensing studies (Smail et al. 1995),

$p\left(z_{\mathrm{s}}\right)=\frac{\beta}{z_{0}^{3} \Gamma(3 / \beta)} z_{\mathrm{s}}^{2} \exp \left[-\left(\frac{z_{\mathrm{s}}}{z_{0}}\right)^{\beta}\right]$

where $\Gamma(x)$ is the complete Euler-gamma function evaluated at $x$, $z_{0}$ is a free parameter that broadly selects the position of the peak, and $\beta$ is another free parameter that defines the extension of the high-redshift tail. Note that, unlike the histograms, this function drops to zero at low redshift. However, the contribution to the global lensing optical depth coming from sources at $z \lesssim 1$ is likely negligible, due to the geometric suppression of lensing efficiency (see also the subsequent discussion in Sect. 5). The resultant best-fitting parameters of these three functions are summarized in Table 1.

The SPZ curve constitutes a good representation of $\mathrm{CH} 05$ data not corrected for spectroscopic incompleteness ${ }^{11}$. The PHZ curve, on the other hand, does not describe the AR07 distribution very well,failing to reproduce its $z \sim 3-4.5$

11 Due to the lack of strong spectral features falling into the observational windows, it is not possible to measure the spectroscopic $z$ of sources in the range $z=1.2-1.8$ (the so called "redshift desert"). 

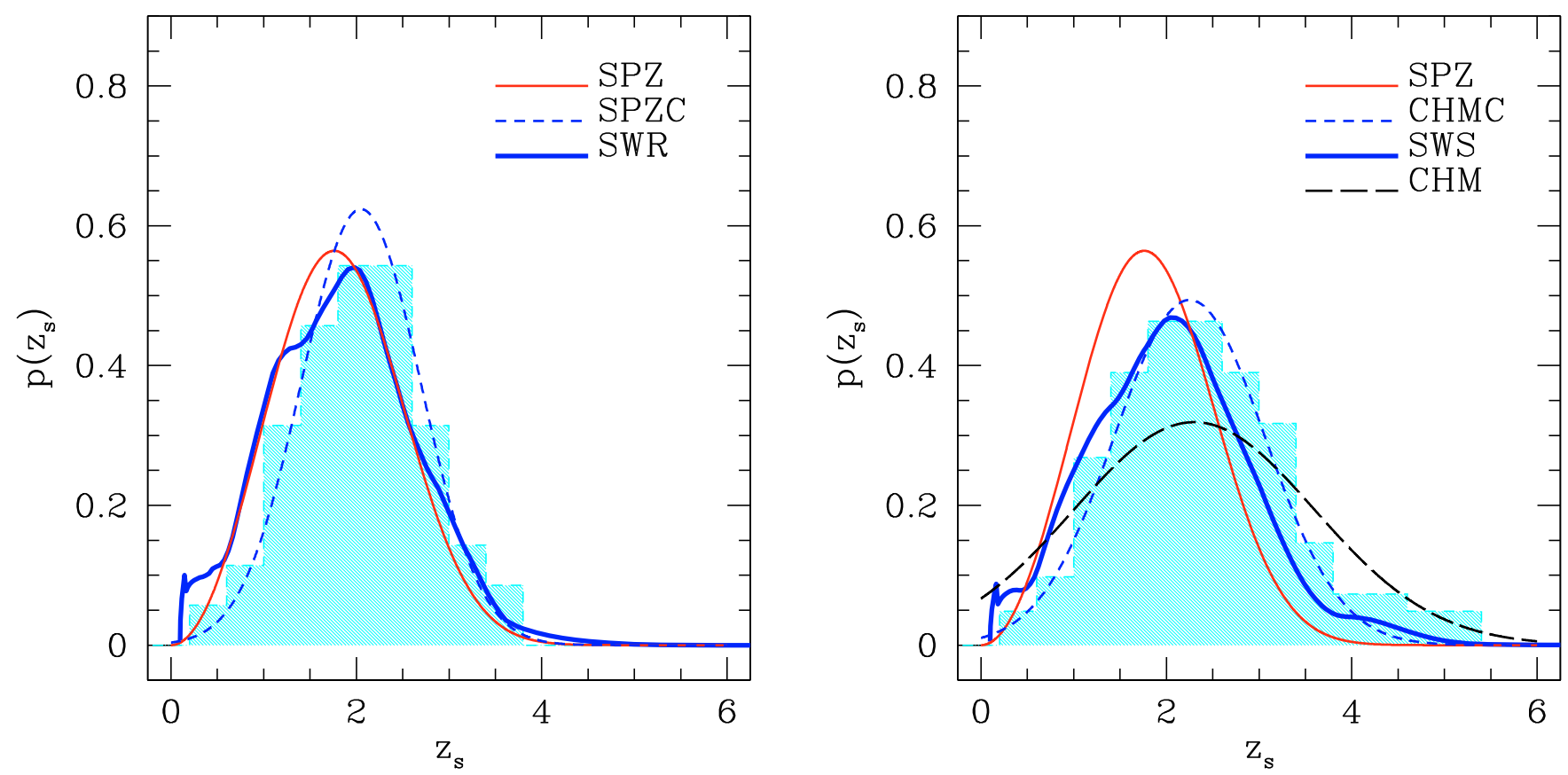

Fig. 4. Left panel. Histogram of the redshift distribution of SMGs derived by Chapman et al. (2005), corrected for spectroscopic incompleteness. This correction was implemented by interpolating the $\mathrm{CH} 05$ distribution in the region of the redshift desert (Swinbank, private communication). The SPZC line indicates the best Gaussian fit to the histogram. The blue solid line corresponds to the redshift distribution of SMGs with $S_{850} \mu \mathrm{m}>$ $5 \mathrm{mJy}$ and $S_{1.4 \mathrm{GHz}}>30 \mu \mathrm{Jy}$ predicted by the semi-analytic model presented in Swinbank et al. (2008). The SPZ curve presented in Fig. 3 has being included for comparison. Right panel. The same histogram, also corrected for radio incompleteness using CH05 evolutionary model. The blue solid line corresponds to the redshift distribution of SMGs with $S_{850 \mu \mathrm{m}}>5 \mathrm{mJy}$ predicted in Swinbank et al. (2008). The CHMC line indicates the best Gaussian fit to the histogram. The SPZ and CHM curves presented in Fig. 3 have being included for comparison.

Table 1. Parameters of the redshift distributions presented in Figs. 3 and 4.

\begin{tabular}{clccc}
\hline \hline Nickname & $z_{0}^{(1)}$ & $\beta^{(1)}$ & $\mathrm{rms}^{(2)}$ & $z_{\mathrm{p}}^{(3)}$ \\
\hline SPZ & 1.99 & 2.81 & - & 1.76 \\
PHZ & 2.51 & 13.3 & - & 2.18 \\
CHM & - & - & 1.30 & 2.30 \\
\hline SPZC $^{(4)}$ & - & - & 0.64 & 2.05 \\
CHMC $^{(4)}$ & - & - & 0.81 & 2.25 \\
\hline
\end{tabular}

(1) Best fit parameters of Eq. (4); (2) width of the best Gaussian fit; (3) peak position of the distribution; (4) the parameters associated with these distributions, provided by M. Swinbank, were misquoted in SW08 and $\mathrm{CH} 05$.

tail. This is a consequence of how the function in Eq. (4) is constructed. In particular, to accommodate the low- $z$ part of the photometric histogram in Fig. 3, the function needs to raise very steeply and hence, by construction, it must also drop steeply at high- $z$. Despite the function in Eq. (4) not being a good choice for fitting the photometric data, we nevertheless included the PHZ curve in our calculations because it highlights the consequence of choosing a distribution that is truncated at $z \sim 3$. Finally, the CHM curve allows us to predict the number of expected arcs if the $\mathrm{CH} 05$ histogram is corrected for spectroscopic incompleteness and high- $z$ SMGs without radio counterparts (radio incompleteness). We stress that at this stage we are not interested in using the best possible representation for the true source redshift distribution, but only to adopt a few motivated choices that broadly cover the range of realistic possibilities, in order to check the corresponding effect on the abundance of arcs.
To show more clearly the different behavior in the highredshift tail of our three choices, we present their cumulative distributions in the right panel of Fig. 3, namely

$P(z)=\int_{0}^{z} p(\xi) \mathrm{d} \xi$

In particular, when $P(z) \simeq 1$ for SPZ, at $z \sim 3.5$, we still have $P(z) \simeq 0.8$ for CHM, implying that $\sim 20 \%$ of the SMGs still can be found at $z \gtrsim 3.5$ using the latter distribution.

In order to further show that this family of three functions cover all the reasonable possibilities, we have compared them with the predictions of one of the semi-analytical models that have been developed to explain the properties of SMGs (see Swinbank et al. 2008, and references therein). The histogram in the left panel of Fig. 4 shows $\mathrm{CH} 05$ data corrected for spectroscopic incompleteness. Note that the SPZ curve is very consistent with the semi-analytic model prediction (SWR), although both curves peak at slightly lower redshift $\left(\Delta z_{p}=0.3\right)$ than the best Gaussian fit of the histogram (SPZC). However, as pointed out in Swinbank et al. (2008, SW08 hereafter), the CH05 distribution is expected to be uncertain by at least $\Delta z \sim 0.25$, which is the field-to-field variation between the seven sub-fields in the CH05 sample due to cosmic variance. Therefore, we can consider SPZ as a good representation of the current observations, despite the fact that it comes from a histogram that was not corrected for spectroscopic incompleteness.

After the computations of the number of arcs were completed, we became aware of the fact that the parameters quoted in $\mathrm{CH} 05$ for the best Gaussian fit to their simple evolutionary model (CHM, see Table 1) were incorrect (Swinbank, private communication). As it is shown in the right panel of Fig. 4, the CHM distribution has a higher- $z$ tail as compared to the correct Gaussian fit (CHMC) and the prediction of the semi-analytical 
model (SWS). Since the true high- $z$ tail of the redshift distribution of SMGs is expected to be in between the cases considered in our calculations (SPZ, PHZ and CHM), and (as it will be discussed in Sect. 5.2) the final effect of the source redshift distribution on the number of arcs is small given the many uncertainties involved, we considered it unnecessary to repeat the calculations for CHMC.

\subsection{Source number counts}

The final ingredient needed to estimate the number of arcs produced by SMGs is the observed surface density of this source population, both at $1.4 \mathrm{GHz}$ and $850 \mu \mathrm{m}$. Let $n_{0}(S)$ be the differential number counts, defined as the surface density of unlensed galaxies per unit flux density $S$. Integrating $n_{0}(S)$ over all fluxes above a given threshold, we obtain the respective cumulative number counts

$N_{0}(S) \equiv \int_{S}^{+\infty} n_{0}(\xi) \mathrm{d} \xi$.

Let $\mu$ be the lensing-induced magnification of images on the lens plane, and $\mu_{+} \equiv|\mu|$. If $P\left(\mu_{+} \mid d_{0}\right)$ is the magnification probability distribution for sources that are imaged into arcs with $d \geq d_{0}$, then the magnified differential number counts can be calculated as (Bartelmann \& Schneider 2001)

$n(S)=\int_{0}^{+\infty} n_{0}\left(\frac{S}{\mu_{+}}\right) \frac{P\left(\mu_{+} \mid d_{0}\right)}{\mu_{+}^{2}} \mathrm{~d} \mu_{+}$.

Hence, the magnified cumulative number counts read as

$N(S) \equiv \int_{S}^{+\infty} n(\xi) \mathrm{d} \xi=\int_{0}^{+\infty} N_{0}\left(\frac{S}{\mu_{+}}\right) \frac{P\left(\mu_{+} \mid d_{0}\right)}{\mu_{+}} \mathrm{d} \mu_{+}$.

As can be seen in Eq. (8), the lensing magnification bias has a twofold effect. On one side, sources that would be too faint to be detected without the action of lensing are amplified, and hence the respective images are brought above the detection threshold. On the other side, the unit solid angle is stretched by lensing magnification, implying that the number density of sources is decreased. Which one of these two effects wins depends on the local slope of the unmagnified cumulative number counts. In particular, if $N_{0}(S) \propto S^{-\alpha}$ and $\alpha>1$, the number density of sources will be increased, while if $\alpha<1$ it will be decreased. It should be noted that, while for the unmagnified counts $N_{0}(S)$ the flux density is derived by integrating the surface brightness over the area of the source, for the magnified counts $N(S)$ the integral is performed over the area of the resulting arc.

Since our main motivation was to provide predictions for the abundance of giant arcs to be detected in surveys carried out with future instruments, we needed to provide the predicted number of arcs as a function of the surface brightness, instead of flux density. The reason is that we are working under the assumption that arcs are resolved structures, and therefore they are observed as extended objects. Under these circumstances, the flux integrated over the resolution element of the instrument (seeing, PSF, pixel or beam) is no longer the total flux of the source (as in the case of unresolved sources), and it may therefore be below the limiting flux although the arc as a whole is not. In other words, arc detectability under these circumstances is not limited by the flux density but rather by the surface brightness.

In order to take this into account, we had to convert the observed number counts as a function of flux density into number counts as a function of surface brightness. Assuming that the size of sources is given by $R_{\mathrm{e}}$, and that the surface brightness is constant across it, then $N_{0}(B)=N_{0}\left(S / \pi R_{\mathrm{e}}^{2}\right)$. Note that, since the surface brightness is not affected by lensing, the magnification bias will manifest itself only through the solid angle stretching. Therefore, the cumulative magnified number counts (as a function of surface brightness) can be written as

$N(B)=N_{0}(B) \int_{0}^{+\infty} \frac{P\left(\mu_{+} \mid d_{0}\right)}{\mu_{+}} \mathrm{d} \mu_{+}$.

Among other things, this implies that the magnification bias will always decrease the cumulative number counts, irrespective of the shape of the unmagnified ones.

In the following we used the magnification distribution given by Fedeli et al. (2008), which is represented by the superposition of two Gaussians. In particular, we adopted the $P\left(\mu_{+} \mid d_{0}\right)$ function for $d_{0}=10$, but the result is virtually the same also for the case $d_{0}=7.5$. Note however, that this (conditional) magnification distribution was computed for a background population of sources that have different morphologies than SMGs (see Sect. 3.1). In principle, the bimodality of the magnification distribution is expected to be preserved because it only depends on the caustic structure ( $\mathrm{Li}$ et al. 2005), but it can be affected by the source morphology in two opposite ways. On one hand, since SMGs are smaller than in Fedeli et al. (2008), we expect large arcs to form closer to the critical curves, and therefore to have larger magnifications on average. On the other hand, the fact that SMGs are more elongated will favor the formation of large arcs in regions of lower magnification. Given the uncertainties in other parts of the calculation, we consider that the use of a magnification distribution derived for optical sources will have a marginal effect on the derived number of arcs produced by SMGs. For a comprehensive review of the many effects that could affect the estimation of arc abundances by galaxy clusters, see the discussion in Fedeli et al. (2008).

\subsubsection{Submm number counts}

For the latest and most complete estimate of the submm number counts at $850 \mu \mathrm{m}$ we refer to Knudsen et al. (2008, hereafter KN08), who carried out a combined analysis of the counts derived from the Leiden SCUBA Lens Survey (LSLS) and the SHADES survey. With an area of $720 \operatorname{arcmin}^{2}$, the SHADES survey is the largest blank-field submm survey completed to date, and therefore the least affected by cosmic variance. It provides the best constraints for the submm number counts in the flux density range $2-15$ mJy (Coppin et al. 2006). On the other hand, the LSLS survey targeted 12 galaxy cluster fields which cover a total area of $71.5 \mathrm{arcmin}^{2}$ in the image plane. It provides the deepest constraints at the faint end of the submm counts $(0.11 \mathrm{mJy}$, after correcting for the lensing magnification). In their analysis, KN08 used two functions to characterize the combined differential number counts from both surveys: a double power-law,

$n_{0}(S)=\frac{n_{0, *} / S_{*}}{\left(S / S_{*}\right)^{\alpha}+\left(S / S_{*}\right)^{\beta}}$

and a Schechter function (Schechter 1976),

$n_{0}(S)=n_{0, *}\left(\frac{S}{S_{*}}\right)^{\alpha+1} \mathrm{e}^{-S / S_{*}}$.

Moreover, when fitting the observed cumulative number counts, they added the supplementary constraint that the integrated light 


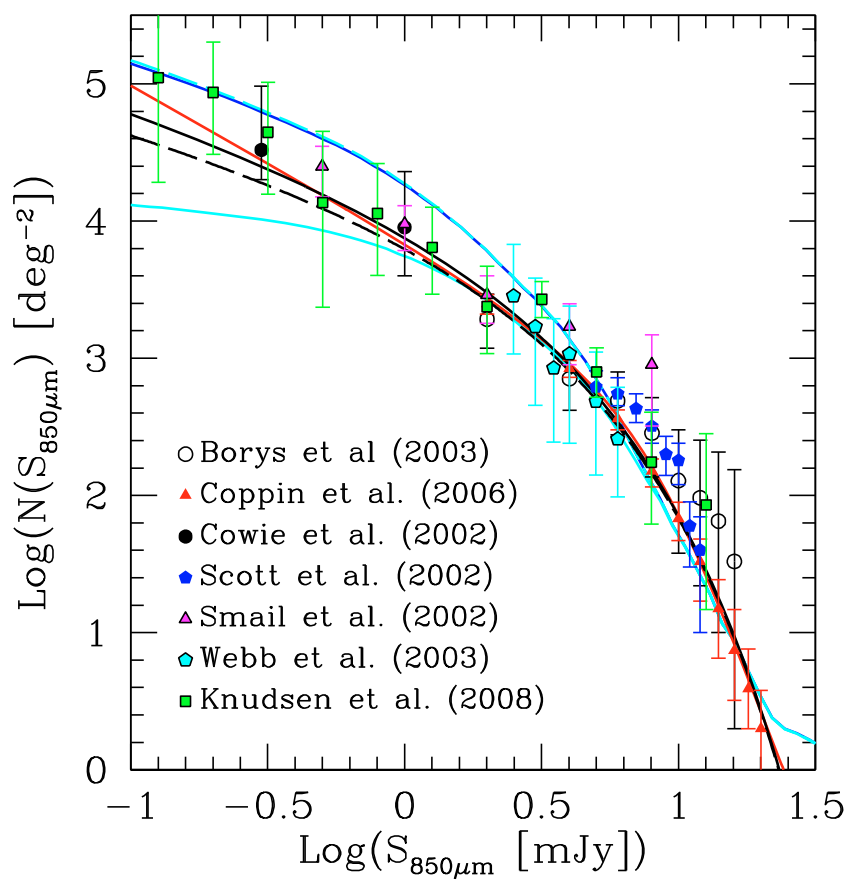

Fig. 5. Comparison between observed and predicted cumulative number counts. The red and black solid curves corresponds to the best-fit double power-law (DB) and the best-fit Schechter function (SB) derived by Knudsen et al. (2008) for the combined $850 \mu$ m cumulative number counts from the LSLS survey (Knudsen et al. 2008) and the SHADES survey (Coppin et al. 2006). The black dashed line indicates the shallowest Schechter function consistent with the data of these two surveys (SM). The blue solid line indicates the cumulative number counts predicted by the semi-analytic model presented in Swinbank et al. (2008) for SMGs with $S_{850 \mu \mathrm{m}}>5 \mathrm{mJy}$. Model predictions for SMGs with $S_{850 \mu \mathrm{m}}>5 \mathrm{mJy}$ and $S_{1.4 \mathrm{GHz}}>30 \mu \mathrm{Jy}$, and SMGs with $S_{850 \mu \mathrm{m}}>5 \mathrm{mJy}$ and $S_{1.4 \mathrm{GHz}}>0.5 \mu \mathrm{Jy}$ are indicated by the cyan solid and dashed lines, respectively. Note that the cyan dashed line and the blue solid line are almost indistinguishable.

well below $0.1 \mathrm{mJy}$ should not be higher than the extragalactic background light (Puget et al. 1996; Fixsen et al. 1998). The resulting best-fit parameters are summarized in Table 2 .

Figure 5 shows the (unmagnified) cumulative number counts derived from the best fit Schechter function (black solid line) and the best fit double power law function (red solid line) presented in KN08 using Eq. (6). Note that, whereas both curves behave almost identically at the bright flux end, their predictions for the low flux number counts differ by a factor of $\sim 2.5$ at $0.1 \mathrm{mJy}$. Since the low flux tail of the submm counts dominates the number of SMGs that could potentially be lensed, we computed predictions for arcs produced by SMGs at $850 \mu \mathrm{m}$ for the two following cases: (i) the shallowest Schechter function consistent with the combined LSLS and SHADES data (also shown in Fig. 5) and (ii) the best fit double power law function, hereafter refered to as SM and DB, respectively. The first one provides the minimum expected number of arcs consistent with observations, whereas the second one gives the number of arcs predicted by the best fit to the data (see Table 2).

The cumulative number counts derived for these two cases as a function of flux density are shown in the top left panel of Fig. 6, including the corresponding counts corrected for magnification bias using Eq. (8). In the same figure, the top right panel shows the cumulative number counts as function of surface brightness. The corresponding counts corrected for
Table 2. Parameters for the $850 \mu \mathrm{m}$ differential number counts.

\begin{tabular}{ccccc}
\hline \hline Name & $S_{*}(\mathrm{mJy})$ & $n_{0, *}^{(4)}$ & $\alpha$ & $\beta$ \\
\hline $\mathrm{DB}^{(1)}$ & $9.6_{-2.12}^{+0.3}$ & $658 \pm 48$ & $2.12_{-0.08}^{+0.14}$ & $6.22_{-0.34}^{+0.51}$ \\
$\mathrm{SB}^{(2)}$ & $4.30 \pm 0.08$ & $1039 \pm 69$ & $-2.62 \pm 0.10$ & - \\
$\mathrm{SM}^{(3)}$ & 4.22 & 970 & -2.52 & - \\
\hline
\end{tabular}

(1) best fit double power-law function, Eq. (10); (2) best fit Schechter function, Eq. (11); ${ }^{(3)}$ shallowest Schechter function consistent with the data; ${ }^{(4)}$ expressed in $\mathrm{deg}^{-2}$ for DB and in $\mathrm{deg}^{-2} \mathrm{mJy}^{-1}$ for SB and SM.

magnification bias (which will be used to compute the number of arcs) were derived using Eq. (9).

\subsubsection{Radio number counts}

Figure 5 also shows the cumulative submm number counts predicted by the SW08 model (blue solid line) compared with the results from different $850 \mu \mathrm{m}$ SCUBA surveys. Note that, although the model tends to over-predict the counts at faint fluxes compared with the best fits provided by KN08 (red and black solid lines), it is consistent with the observational errors. The cyan solid line indicates the predicted counts for SMGs with radio counterparts assuming $S_{1.4 \mathrm{GHz}}>30 \mu \mathrm{Jy}$. The fact that its shape is different from the shape of the blue solid curve is because current observations only detect radio emission from $\sim 60 \%$ of the observed SMGs. However, if we allow the sensitivity threshold to go down to the $\mu \mathrm{Jy}$ level expected for $e$-MERLIN, the SW08 model indicates that it would be possible to detect all the radio counterparts of SMGs with $S_{850 \mu \mathrm{m}}>$ 5 mJy (cyan dashed line).

Since SMGs seem to follow the FIR/radio correlation (e.g. Kovács et al. 2006), the $1.4 \mathrm{GHz}$ number counts of SMGs (which we need to predict the number of radio arcs) could be derived by scaling the $850 \mu \mathrm{m}$ number counts introduced in the previous section (DB and SM). As shown in Fig. 7 of $\mathrm{CH} 05$, the ratio between the $850 \mu \mathrm{m}$ flux density and the $1.4 \mathrm{GHz}$ flux density shows a broad scatter (up to one order of magnitude), which is probably a consequence of the strong influence of the dust temperature on the SEDs of SMGs. However, most of the points in this figure are located between redshift 2 and 3, and have an average $S_{850 \mu \mathrm{m}} / S_{1.4 \mathrm{GHz}}$ ratio between 50 and 100 . Therefore, we decided to use these two scaling factors to derive first order upper and lower limits of the radio number counts of SMGs. The resultant cumulative radio number counts are shown in the lower panels of Fig. 6. Note that the values of 50 and 100 chosen for the submm/radio flux density ratio are meant to be indicative, since there are still many sources that display a ratio below 50 or above 100. The reader interested in results given by different values of this ratio can scale the curves appropriately in the upper panels of Fig. 6. Also, exact numerical values can be made available by the authors upon request.

\section{Strong lensing optical depth}

To compute the total optical depth for lensed SMGs, we constructed a synthetic cluster population composed of $q=$ 1000 cluster-sized dark-matter halos with masses uniformly distributed in the interval $\left[10^{14}, 2.5 \times 10^{15}\right] M_{\odot} h^{-1}$ at $z=0$. Note that it is not necessary to extract these masses according to the cluster mass function, since this is already taken into account in Eq. (1) by weighting the cross sections with the function $n(M, z)$. 

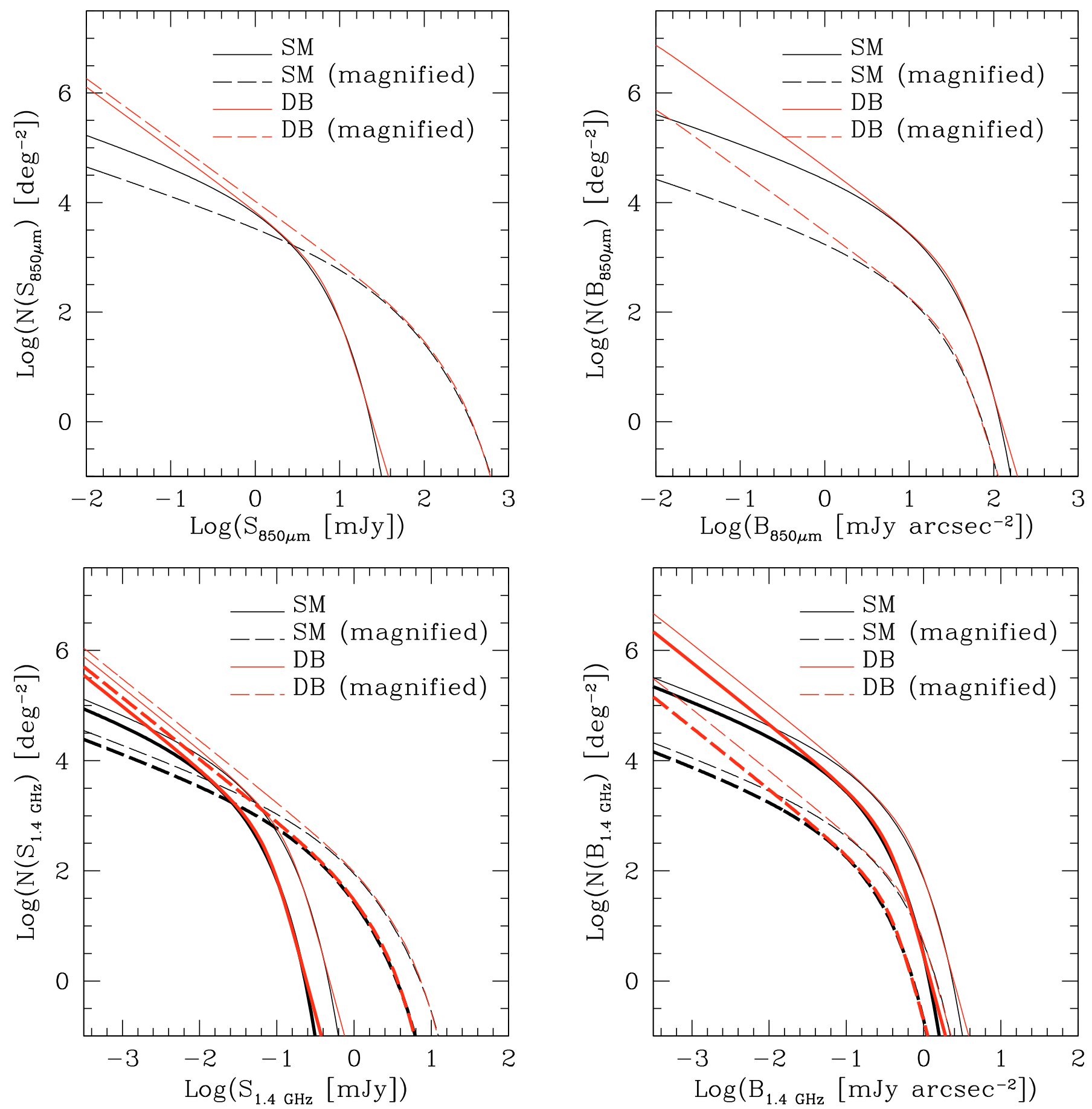

Fig. 6. The cumulative number counts of SMGs at $850 \mu \mathrm{m}$ (top panels) and $1.4 \mathrm{GHz}$ (bottom panels), as a function of flux density (left panels) and surface brightness (right panels). The conversion from flux density into surface brightness was done assuming that the emission at both submm and radio wavelengths is homogeneous and have $R_{\mathrm{e}}=0.25^{\prime \prime}$. Thin lines correspond to $S_{1.4 \mathrm{GHz}}=S_{850 \mu \mathrm{m}} / 50$, while thick lines assume $S_{1.4 \mathrm{GHz}}=S_{850 \mu \mathrm{m}} / 100$. The magnification pattern used to compute the magnified number counts is the one for arcs with $d \geq d_{0}=10$. SM indicates the shallowest Schechter function that is consistent with the data, while DB represents the best fit double power-law (see the text for more details).

The structure of each cluster is modeled using the NFW density profile (Navarro et al. 1995, 1996, 1997), which constitutes a good representation of average dark-matter halos over a wide range of masses, redshifts and cosmologies in numerical $n$-body simulations (Dolag et al. 2004). Several studies of strong lensing and X-ray luminous clusters also show that these are well fitted by an NFW profile (Schmidt \& Allen 2007; Oguri et al. 2009). This profile also has the advantage that its lensing properties can be described analytically (Bartelmann 1996).
To account for the asymmetries of real galaxy clusters, the halos are assumed to have elliptically distorted lensing potentials. However, instead of considering a single ellipticity value to describe all the synthetic cluster lenses, we derived an ellipticity distribution from a set of numerical clusters extracted from the MareNostrum simulation (Gottlöber \& Yepes 2007). The strong lensing analysis required to generate this ellipticity distribution was taken from Fedeli et al. (2009, in preparation), as described in Sect. 3.1. For each simulated cluster, the 


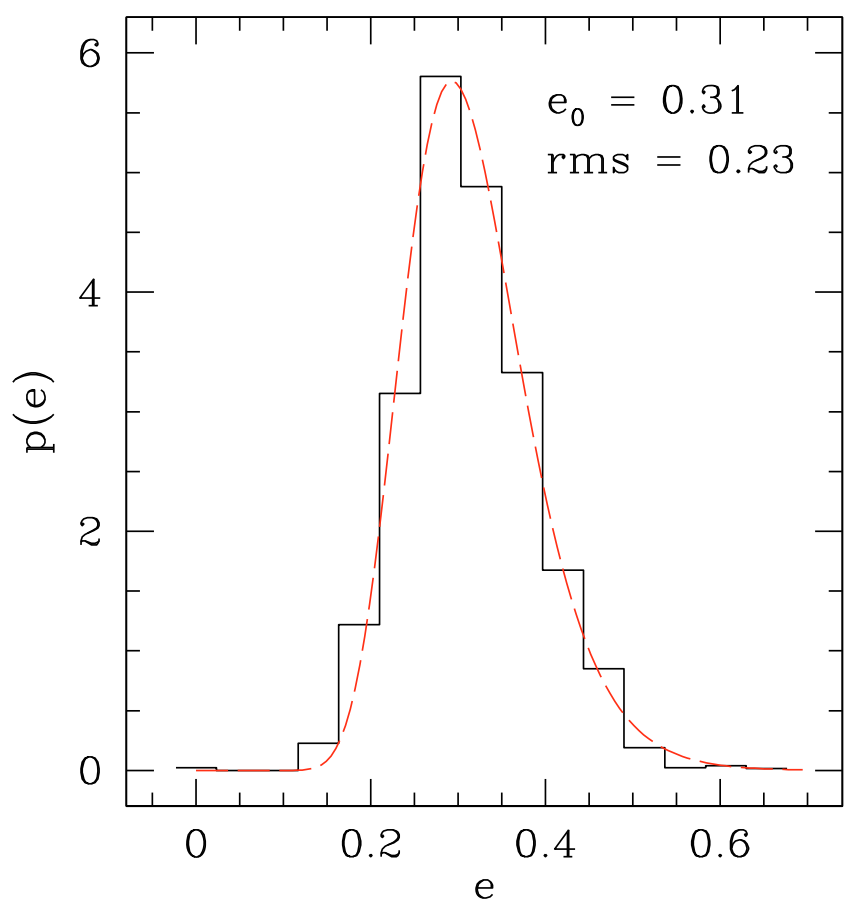

Fig. 7. The distribution of NFW lens ellipticities fitting the cross sections of a sample of numerical clusters. The red dashed line represents the best fit log-normal distribution, whose median and dispersion are labeled in the top-right corner of the plot.

lensing analysis along three orthogonal projections was performed, computing the cross sections for arcs with $d \geq d_{0}=7.5$ and sources with $z_{\mathrm{s}}=2$. For each of these projections, we found the ellipticity $e$ of the NFW lens whose cross section is closest to the cross section of the numerical cluster, i.e., we found the ellipticity that minimizes the quantity

$r(e)=\left|\sigma_{7.5}^{(\mathrm{n})}-\sigma_{7.5}(e)\right|$,

where $\sigma_{7.5}^{(\mathrm{n})}$ is the cross section of the numerical lens, and $\sigma_{7.5}(e)$ is that of the NFW lens for a given potential ellipticity $e$.

Figure 7 shows the distribution of the ellipticities that minimize the quantity $r(e)$ in Eq. (12) for all clusters in our numerical analysis. The dashed red line is derived by fitting the distribution with a log-normal function of the kind

$p(e) \mathrm{d} e=\frac{1}{\sqrt{2 \pi} \sigma_{\mathrm{e}}} \exp \left[-\frac{\left[\ln (e)-\ln \left(e_{0}\right)\right]^{2}}{2 \sigma_{\mathrm{e}}^{2}}\right] \mathrm{d} \ln (e)$,

where the best-fit parameters are $e_{0}=0.31$ and $\sigma_{\mathrm{e}}=0.23$. The ellipticity values used to characterize the potential of the synthetic NFW cluster lenses were then randomly extracted from the above distribution.

Elliptical NFW profiles are a good representation of realistic cluster lenses only when the clusters do not undergo major merger events (Meneghetti et al. 2003). Since the merger activity of galaxy clusters is known to have a significant effect on the statistics of giant arcs (Torri et al. 2004; Fedeli et al. 2006), it has to be taken into account in the construction of the synthetic cluster population. For this reason, we used the excursion set formalism $^{12}$ developed by Lacey \& Cole (1993) (see also Bond et al. 1991; Somerville \& Kolatt 1999) to construct a backward

\footnotetext{
12 Also referred to as the "extended Press \& Schechter (1974) formalism".
}

merger tree for each model cluster at $z=0$, assuming that each merger is binary (see discussion in Fedeli \& Bartelmann 2007). When a merger happens, the event is modeled assuming that the two merging halos (also described as elliptical NFW density profiles) approach each other at a constant speed. The duration of the merger is set by the dynamical timescales of the two halos (see Fedeli \& Bartelmann 2007; and Fedeli et al. 2008, for a detailed description of the modeling procedure).

With the synthetic cluster population constructed in this way, the total average optical depth was derived by computing individual cross sections with the semi-analytic algorithm developed by Fedeli et al. (2006), especially designed to estimate the strong lensing cross sections of individual lenses in a fast and reliable way. The optical depth for a discrete set of lenses can be recast as

$\tau_{d_{0}}\left(z_{\mathrm{s}}\right)=\frac{1}{4 \pi D_{\mathrm{s}}^{2}} \int_{0}^{z_{\mathrm{s}}}\left[\sum_{i=1}^{q-1} \bar{\sigma}_{d_{0}, i}(z) \int_{M_{i}}^{M_{i+1}} n(M, z) \mathrm{d} M\right] \mathrm{d} z$

where the masses $M_{i}(1 \leq i \neq q)$ have to be sorted from the lowest to the highest at each redshift step, and the quantity $\bar{\sigma}_{d_{0}, i}(z)$ is defined as

$\bar{\sigma}_{d_{0}, i}(z) \equiv \frac{1}{2}\left[\sigma_{d_{0}}\left(M_{i}, z\right)+\sigma_{d_{0}}\left(M_{i+1}, z\right)\right]$.

This effectively means that, for all the clusters with mass between $M_{i}$ and $M_{i+1}$, we assume the average cross section of the model dark-matter halos with masses $M_{i}$ and $M_{i+1}$. The algorithm of Fedeli et al. (2006) for computing strong lensing cross sections consists of first assuming sources as point-like circles, and then introducing the effect of source ellipticity according to Keeton (2001). The source finite size is taken into account by convolving the local lensing properties over the typical source size.

The total average optical depth is calculated by integrating Eq. (14) over the source redshift distribution. Effectively, the $p\left(z_{\mathrm{s}}\right)$ weighting is avoided since we assigned individual source redshifts (randomly extracted from the distribution $p\left(z_{\mathrm{s}}\right)$ ) at each of the $q$ halos in the cluster sample and evolved their merger trees back in time until the respective source redshift. Given the large number of synthetic dark-matter halos used, this approach allows one to omit $p\left(z_{\mathrm{s}}\right)$ in Eq. (2) when the redshift integral is discretized.

Despite the fact that the ellipticity distribution used to model the synthetic cluster population was derived for $d \geq d_{0}=7.5$, the best fit parameters of Eq. (13) can be used to compute the cross sections for arcs also with $d_{0}=10$ without compromising the results. The reason is that, although there might be a mild dependence of the distribution of lensing ellipticities on $d_{0}$, it is the overall caustic structure that defines the abundance of arcs above a certain $d_{0}$, regardless of its precise value. In fact, the criterion used to determine the ellipticity distribution is based on the similarity between the cross sections of the NFW lens and the numerical lens, which is an indirect way of comparing the overall caustic structures produced by both kinds of lenses. To verify this argument, the ellipticity distribution was re-computed using a criterion that is directly related to the caustic structure, that is, by defining the best fitting ellipticity as the one that minimizes the modified Hausdorff distance ${ }^{13}$ between the critical lines of

13 This parameter constitutes one of the best ways to quantify the morphological difference between two sets of points (Dubuisson \& Jain 1994). See also Rzepecki et al. (2007) for a different application to gravitational lensing. 

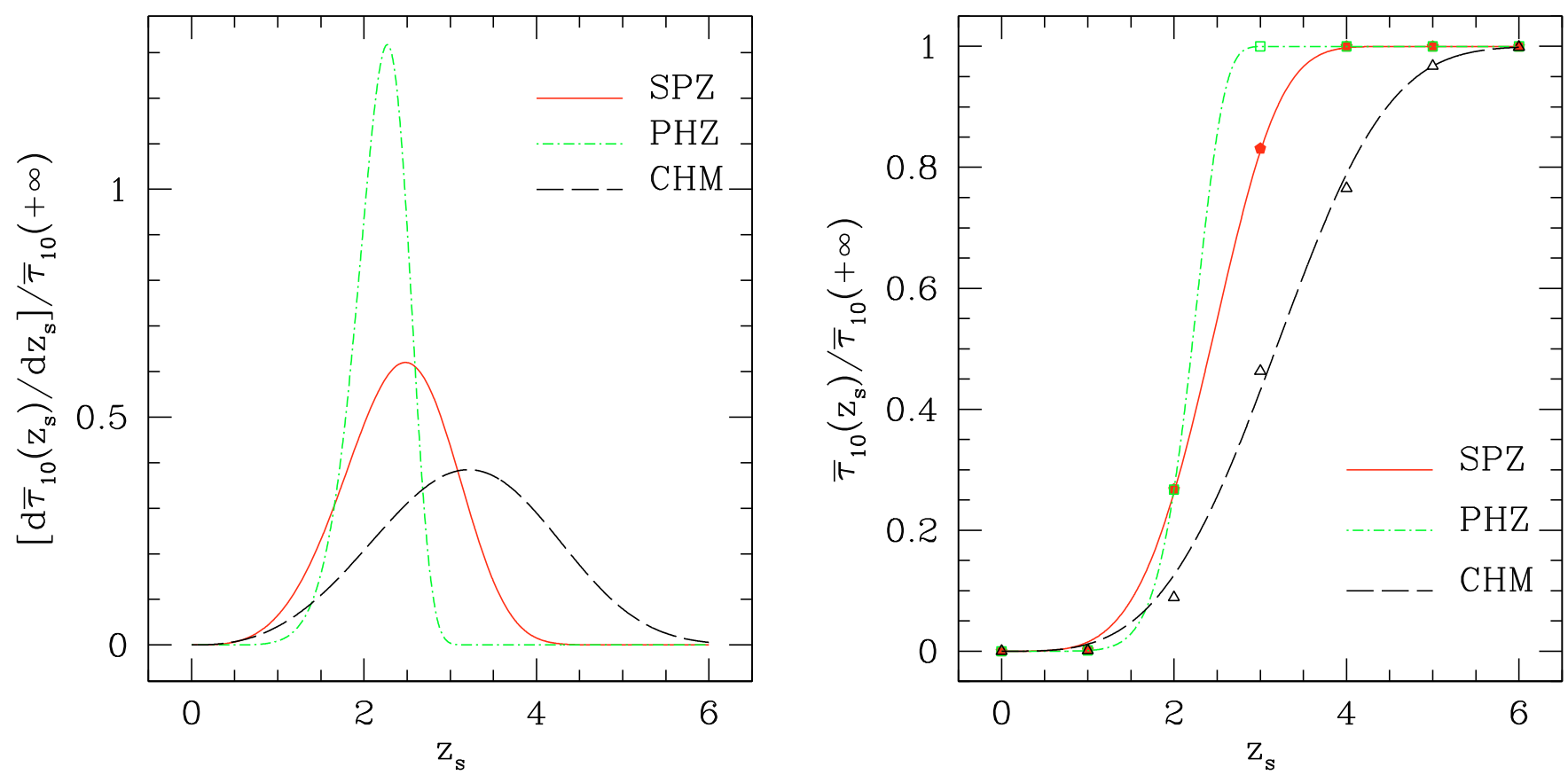

Fig. 8. Left panel: differential redshift distributions of SMGs producing large gravitational arcs, corresponding to the cumulative distributions reported in the right panel. Right panel: the cumulative redshift distributions of SMGs producing radio arcs with $d \geq d_{0}=10$ for input source redshift distributions SPZ, PHZ and CHM (see the text for more details). Lines are the best fit functions given in Eq. (16) and Table 3, according to the labels in the plot.

the numerical and NFW lenses. The ellipticity distribution obtained in this way is very similar to the one depicted in Fig. 7 $\left(e_{0}=0.30, \mathrm{rms}=0.34\right)$.

The median of the ellipticity distribution derived in this work using the lensing cross section $\left(e_{0}=0.31\right.$, see Fig. 7) is fully consistent with the one obtained by Meneghetti et al. (2003) comparing the deflection angle maps $(e \sim 0.3$, using lenses placed at $z \sim 0.3$ and a source population at $z_{\mathrm{s}}=1$ ). Even though these two criteria are arguably tightly related, the former is a quantity that is more directly related to observables than the latter, hence it is reassuring that they give comparable results. In addition, this result extends the previous one by quantifying the scatter around the median ellipticity and dealing with lenses that are distributed in redshift.

\section{Results}

\subsection{Arc redshift distribution}

The redshift distribution of arcs with $d \geq d_{0}$ (arc redshift distribution hereafter) is expected to provide information about the redshift distribution of the background source population that is being lensed. However, it will also be distorted by the fact that the abundance of massive and compact galaxy clusters evolves with redshift, and that the lensing efficiency depends on the relative distances of sources and lens with respect to the observer.

To assess the potential of this approach to gather information about the intrinsic redshift distribution of SMGs, we derived the arc redshift distribution associated with each of the three source redshift distributions used as inputs in our calculations (see Fig. 3). This was done by computing, for each input distribution, the average optical depth $\bar{\tau}_{d_{0}}\left(z_{\mathrm{s}}\right)$ for several different values of $z_{\mathrm{s}}$. That is, we excluded in the computation of the average optical depth those model clusters (and their respective chain of progenitors) whose associated source redshifts were $>z_{\mathrm{s}}$. The resultant cumulative arc redshift distributions, obtained after
Table 3. Parameters of the arc redshift distributions shown in Fig. 8.

\begin{tabular}{ccc}
\hline \hline$p\left(z_{\mathrm{s}}\right)$ & $z_{\mathrm{s}, *}$ & $\gamma$ \\
\hline SPZ & 2.64 & 4.31 \\
PHZ & 2.31 & 8.20 \\
CHM & 3.53 & 3.53 \\
\hline
\end{tabular}

normalizing the optical depth for each $z_{\mathrm{s}}$ to the total average optical depth $\bar{\tau}_{d_{0}}(+\infty)=\bar{\tau}_{d_{0}}$, are shown in the right panel of Fig. 8 . The lines correspond to the best fit for each distribution provided by the simple function

$\frac{\bar{\tau}_{d_{0}}\left(z_{\mathrm{s}}\right)}{\bar{\tau}_{d_{0}}(+\infty)}=1-\exp \left(-\frac{z_{\mathrm{s}}}{z_{\mathrm{s}, *}}\right)^{\gamma}$

where $z_{\mathrm{s}, *}$ indicates where the transition between the extrema 0 and 1 occur, and $\gamma$ indicates how sharp this transition is. The best-fit parameters for each of the three input source redshift distributions are summarized in Table 3. The corresponding differential arc redshift distributions are shown in the left panel of Fig. 8.

Once more, these arc redshift distributions have been shown for arcs with length-to-width ratio larger than $d_{0}=10$ only. As we verified, since the relative contribution of individual model clusters to the total average optical depth is the same for both $d_{0}=7.5$ and $d_{0}=10$, the resulting arc redshift distribution also does not change significantly between the two choices.

A comparison between Figs. 8 and 3 shows that, as expected, the arc redshift distributions reflect the general properties of the source redshift distributions used as input, but there are also some noteworthy differences between them. For instance, the arc redshift distribution associated with $\mathrm{CHM}$ tends to zero at very low redshift, unlike in the case of the original CHM distribution. The reason is that low redshift sources do not produce many arcs, because (i) they have very few potential lenses at their disposal; 
and (ii) the lensing efficiency of those lenses is very low due to geometric suppression. This results in a lack of low redshift arcs in the distribution, which shifts its peak to higher redshifts compared with the CHM peak (from $z_{\mathrm{p}}=2.3$ to $z_{\mathrm{p}} \sim 3.2$ ). Similarly, the peak of the arc redshift distribution corresponding to SPZ is shifted from $z_{\mathrm{p}}=1.76$ to $z_{\mathrm{p}} \gtrsim 2$.

On the other hand, the arc redshift distribution corresponding to PHZ is not significantly shifted but visibly narrowed. As in the previous cases, low-redshift sources are removed from the distribution because they cannot produce arcs, but the distribution could not shift at higher redshift because the input source redshift distribution is immediately truncated at $z_{\mathrm{s}} \lesssim 3$. In other words, there is too little room between the drop due to lensing efficiency and the one due to the cutoff of the input distribution to allow a significant shift in its peak, and the only possible consequence for the distribution is to shrink and increase the peak height in order to preserve the normalization.

In general, it is apparent that the differences between different source redshift distributions are somewhat enhanced when it comes to the arc redshift distribution. Therefore, assuming that redshift information is available for arcs, this approach can in principle be used to obtain some information about the general characteristics of the source redshift distribution, although it will probably not allow one to distinguish between redshift distributions that are very similar.

\subsection{Number of radio and submm arcs}

In this section we present and discuss the main results of this work: the predicted number of arcs produced by SMGs at radio and submm wavelengths. To that end, we computed the total average optical depth for each of the three source redshift distributions presented in Sect. 3.2 (PHZ, SPZ and CHM), and for arcs with length-to-width ratio higher than both $d_{0}=7.5$ and $d_{0}=10$. These quantities were then multiplied by the magnified cumulative source number counts presented in Sect. 3.3 (SM and DB), to obtain the arc number counts as function of surface brightness. The results, extrapolated to the whole sky, are shown in Fig. 9. A detailed list with the predicted number of submm and radio arcs for different sensitivities is presented in Tables 4 and 6, respectively.

Note that the arc number counts given by the source redshift distributions SPZ and PHZ are almost indistinguishable on the scale of Fig. 9, irrespective of the length-to-width threshold $d_{0}$ adopted. As expected, SPZ produces more large arcs than PHZ because it peaks at higher redshift, but only by a factor of $\sim 7 \%$. The redshift distribution CHM, on the other hand, produces more large arcs than the other two by a factor of $\sim 2$. Since, as mentioned in Sect. 3.2, the distributions CHM and PHZ can be considered as upper and lower limits to the true redshift distribution respectively, we can conclude that the uncertainty introduced by the redshift distribution in the predicted number of arcs is less than a factor of two.

In terms of the length-to-width threshold, the number of arcs predicted for $d_{0}=7.5$ is larger than for $d_{0}=10$ (as was also expected). However, the ratio between the number of arcs with $d \geq d_{0}=7.5$ and $d \geq d_{0}=10$ is not exactly equal to the ratio in the respective optical depths, since the magnification distributions for the two kinds of arcs are also different (see the discussion in Fedeli et al. 2008).

Finally, when it comes to comparing the results from the two adopted source number counts (DB and SM), we see that the difference in the predicted number of arcs is negligible for submm surface brightness limits greater than $5 \mathrm{mJy} \operatorname{arcsec}^{-2}$.
However, at $B_{850 \mu \mathrm{m}}=0.5 \mathrm{mJy} \operatorname{arcsec}^{-2}$, the function DB predicts 2 times more arcs than SM, and the difference becomes a factor of 5 for $0.1 \mathrm{mJy} \operatorname{arcsec}^{-2}$. In the radio domain, the difference between DB and $\mathrm{SM}$ is negligible for $B_{1.4 \mathrm{GHz}}=$ $50 \mu \mathrm{Jy} \operatorname{arcsec}^{-2}$, a factor $\sim 2$ for $10 \mu \mathrm{Jy} \operatorname{arcsec}^{-2}$, a factor $\sim 3$ for $5 \mu \mathrm{Jy} \operatorname{arcsec}^{-2}$ and a factor $\sim 8$ for $1 \mu \mathrm{Jy} \operatorname{arcsec}^{-2}$. Therefore, the uncertainty in the predicted number of arcs is clearly dominated by the uncertainty of the source number counts at the faint surface brightness end.

Considering an all-sky submm survey with enough resolution to resolve individual arcs with $d \geq d_{0}=7.5\left(\sim 0.2^{\prime \prime}\right)$, and a sensitivity of $B_{850 \mu \mathrm{m}}=1 \mathrm{mJy} \operatorname{arcsec}^{-2}$, our calculations predict between $\sim 500$ (PHZ SM) and $\sim 600$ (SPZ DB) arcs with a signalto-noise ratio (SNR) larger than 5 . In the case of $d \geq d_{0}=10$, the expected number of arcs would be between 200 and 250. If the sensitivity is reduced to $0.1 \mathrm{mJy} \operatorname{arcsec}^{-2}$, these predictions can vary between $\sim 3400$ and $\sim 8300$ for $d_{0}=7.5$, and between 1400 and 3600 for $d_{0}=10$.

In a similar way, an all sky radio survey with a sensitivity of $B_{1.4 \mathrm{GHz}}=0.1 \mathrm{mJy} \operatorname{arcsec}^{-2}$ would detect between 8 and 50 arcs for $d_{0}=7.5$ (between none and 25 for $d_{0}=10$ ) at $S N R \geq$ 5 , with the main uncertainty given by the $S_{850 \mu} / S_{1.4 \mathrm{GHz}}$ ratio used to obtain the radio number counts by scaling the observed submm counts. If the limiting radio surface brightness is reduced to $\sim 10 \mu \mathrm{Jy} \operatorname{arcsec}^{-2}$, the predicted number of arcs could be increased to $\sim 500-1300$ for $d_{0}=7.5$ (200-600 for $\left.d_{0}=10\right)$. In order to have excellent statistics with a few thousand giant arcs with $S N R \geq 5$, it would be necessary to go as deep as $S_{1.4 \mathrm{GHz}}=1 \mu \mathrm{Jy} \operatorname{arcsec}^{-2}$. The largest number of arcs is given by the CHM source redshift distribution, which is about a factor of 2 larger than the number of arcs predicted by SPZ and PHZ.

It is plausible that future radio and submillimeter surveys of galaxy clusters would focus on the most massive objects, since the center of attention of many multiwavelength studies is on X-ray bright clusters. To roughly evaluate the effect of this kind of selection, we re-computed the optical depths by including only those clusters in our synthetic population with mass $M \geq 5 \times 10^{14} M_{\odot} h^{-1}$. The resulting arc number counts are presented in Fig. 10, where we used the same scale and line types as in Fig. 9 to ease comparison. The corresponding numbers of submm and radio arcs predicted for different sensitivities are listed in Tables 5 and 7, respectively. The most interesting feature about these new plots is that the relative difference in the arc number counts produced by the CHM distribution on the one hand, and PHZ and SPZ on the other, is significantly reduced. This is due to the fact that massive clusters are found mainly at low redshift, hence lower-mass higher-redshift lenses, that are accessible only to the CHM distribution, become unimportant.

The order-of-magnitude reduction in the abundance of giant arcs when focusing only on clusters with mass $M \geq 5 \times$ $10^{14} M_{\odot} h^{-1}$ is in agreement with the fact that the bulk of the lensing signal actually comes from low-mass clusters, since the optical depth is obviously dominated by the lowest mass objects that are capable of producing a non-vanishing cross sections (see Eq. (1)). Note also that, unlike the previous case, PHZ produces slightly more arcs than SPZ. This is due to the fact that we are including in the calculations only low- $z$ clusters, and PHZ actually has more sources with, e.g., $z_{\mathrm{s}}>1$ than SPZ (see Fig. 3).

Considering again an all-sky submm survey with enough resolution to resolve individual arcs with $d_{0}=7.5$ and sensitivity of $B_{850 \mu \mathrm{m}}=1 \mathrm{mJy} \operatorname{arcsec}^{-2}$, we predict $\sim 20 \operatorname{arcs}$ with $S N R \geq 5$ (8 if $d_{0}=10$ ). This number can increase to 100-250 (40-90) if $B_{850 \mu \mathrm{m}} \geq 0.1 \mathrm{mJy} \operatorname{arcsec}^{-2}$. In the case of a radio survey, it would be necessary to push the limiting flux density down to 

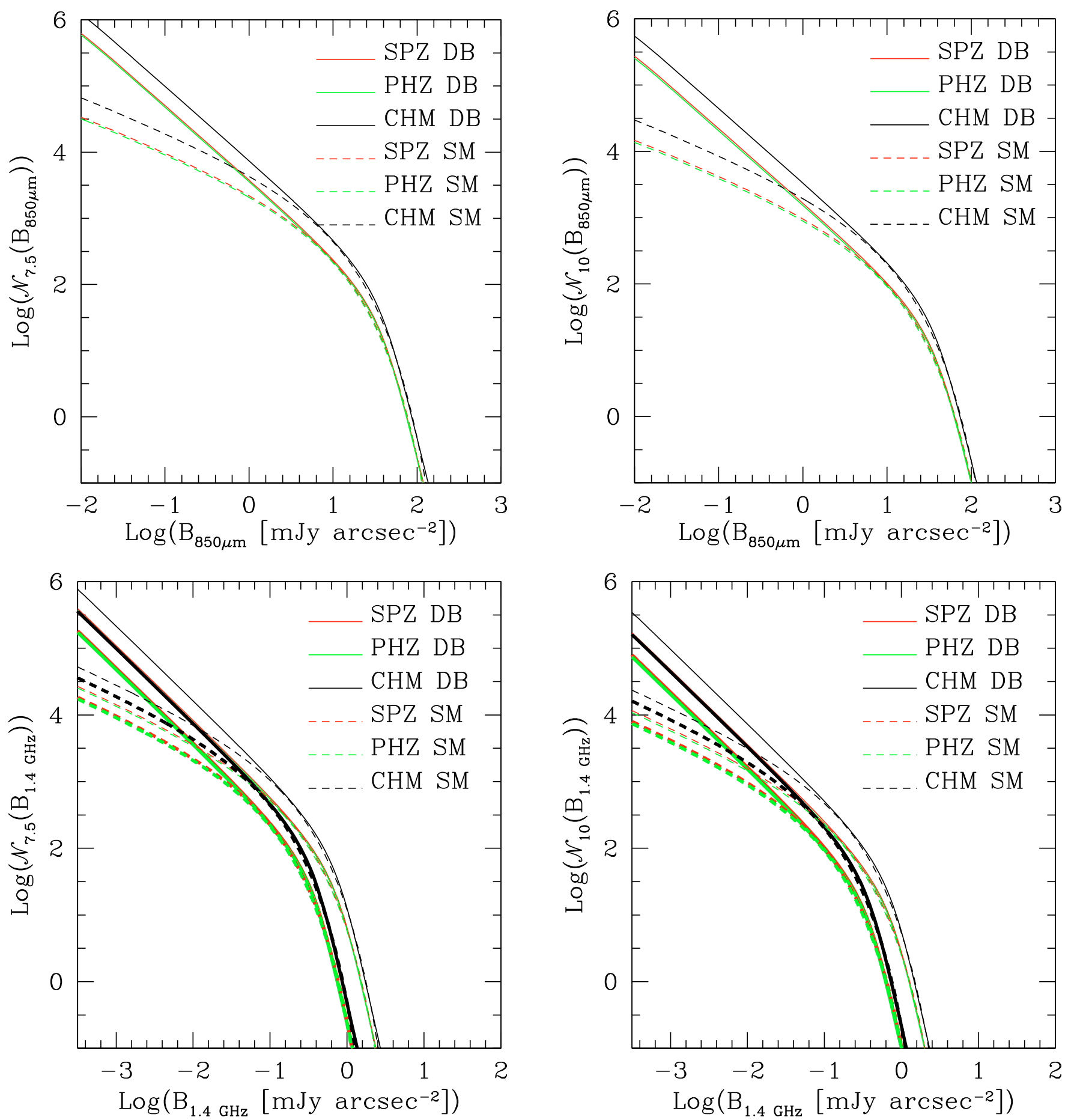

Fig. 9. The total number of arcs with $d \geq d_{0}=7.5$ (left panels) and $d \geq d_{0}=10$ (right panels) that are predicted to be observed in the whole sky above the surface brightness reported on the abscissa. Results for each of the three input source redshift distributions as well as both source number counts adopted in this work are shown, according to the labels. The two top panels refer to surface brightness at $850 \mu \mathrm{m}$, while the bottom panels refer to $1.4 \mathrm{GHz}$. In the bottom panels, thin lines refer to $S_{1.4 \mathrm{GHz}}=S_{850 \mu \mathrm{m}} / 50$, while thick lines refer to $S_{1.4 \mathrm{GHz}}=S_{850 \mu \mathrm{m}} / 100$. Please note the difference in scale on the abscissa between top and bottom panels.

$\sim 1 \mu \mathrm{Jy} \operatorname{arcsec}^{-2}$ to detect few hundred arcs (between 40-200 if $d_{0}=10$ ).

\section{Comparison with previous work}

The probability of strong gravitational lensing due to background sources at radio and submm wavelengths has been a poorly studied issue in the past years. In addition, the few works available in the literature usually involve cosmological models, deflector mass ranges, and modeling approaches for the source and lens populations that are different from the ones used in the present work, making the comparison between them difficult and often not possible. With this note of caution, we tried however to make some of these tentative comparisons in the following. This required us to repeat the calculations in the last section using the number counts as a function of flux density instead of surface 
Table 4. Predicted number of submm $(850 \mu \mathrm{m})$ arcs produced by SMGs for an all-sky survey, using all the synthetic cluster population to compute the optical depths (see Fig. 9).

\begin{tabular}{|c|c|c|c|c|c|c|c|c|}
\hline \multirow{2}{*}{$\begin{array}{c}B_{850 \mu \mathrm{m}}^{(1)} \\
d_{0}^{(2)}\end{array}$} & \multicolumn{2}{|c|}{$5 \mathrm{mJy} \operatorname{arcsec}^{-2}$} & \multicolumn{2}{|c|}{$1 \mathrm{mJy} \operatorname{arcsec}^{-2}$} & \multicolumn{2}{|c|}{$0.5 \mathrm{mJy} \operatorname{arcsec}^{-2}$} & \multicolumn{2}{|c|}{$0.1 \mathrm{mJy} \operatorname{arcsec}^{-2}$} \\
\hline & 7.5 & 10 & 7.5 & 10 & 7.5 & 10 & 7.5 & 10 \\
\hline $\mathrm{SPZ} \mathrm{DB}^{(3)}$ & 575 & 250 & 3792 & 1650 & 8275 & 3600 & 50767 & 22075 \\
\hline PHZ DB ${ }^{(3)}$ & 550 & 233 & 3575 & 1533 & 7800 & 3342 & 47892 & 20517 \\
\hline $\mathrm{CHM} \mathrm{DB}^{(3)}$ & 1142 & 517 & 7425 & 3325 & 16200 & 7267 & 99458 & 44592 \\
\hline $\mathrm{SPZ} \mathrm{SM}^{(3)}$ & 542 & 233 & 2208 & 958 & 3567 & 1550 & 9658 & 4200 \\
\hline PHZ SM $^{(3)}$ & 508 & 217 & 2083 & 892 & 3367 & 1442 & 9117 & 3900 \\
\hline $\mathrm{CHM} \mathrm{SM}^{(3)}$ & 1058 & 475 & 4317 & 1933 & 6992 & 3133 & 1058 & 475 \\
\hline
\end{tabular}

(1) Surface brightness limit used to determine the number of arcs; ${ }^{(2)}$ arc length-to-width ratio thresholds; ${ }^{(3)}$ redshift distribution and number count function, as introduced in Figs. 3 and 6.

Table 5. Predicted number of submm $(850 \mu \mathrm{m})$ arcs produced by SMGs for an all-sky survey, using only clusters with $M \geq 5 \times 10^{14} M_{\odot} h^{-1}$ to compute the optical depths (see Fig. 10).

\begin{tabular}{|c|c|c|c|c|c|c|c|c|}
\hline \multirow{2}{*}{$\begin{array}{c}B_{850 \mu \mathrm{m}}^{(1)} \\
d_{0}^{(2)}\end{array}$} & \multicolumn{2}{|c|}{$5 \mathrm{mJy} \operatorname{arcsec}^{-2}$} & \multicolumn{2}{|c|}{$1 \mathrm{mJy} \operatorname{arcsec}^{-2}$} & \multicolumn{2}{|c|}{$0.5 \mathrm{mJy} \operatorname{arcsec}^{-2}$} & \multicolumn{2}{|c|}{$0.1 \mathrm{mJy} \operatorname{arcsec}^{-2}$} \\
\hline & 7.5 & 10 & 7.5 & 10 & 7.5 & 10 & 7.5 & 10 \\
\hline $\mathrm{SPZ} \mathrm{DB}^{(3)}$ & 17 & 8 & 125 & 42 & 267 & 92 & 1633 & 550 \\
\hline PHZ DB ${ }^{(3)}$ & 17 & 8 & 133 & 42 & 283 & 92 & 1733 & 583 \\
\hline $\mathrm{CHM} \mathrm{DB}^{(3)}$ & 25 & 8 & 150 & 50 & 325 & 108 & 2008 & 683 \\
\hline SPZ SM $^{(3)}$ & 17 & 8 & 75 & 25 & 117 & 42 & 308 & 108 \\
\hline PHZ SM ${ }^{(3)}$ & 17 & 8 & 75 & 25 & 125 & 42 & 333 & 108 \\
\hline $\mathrm{CHM} \mathrm{SM}^{(3)}$ & 25 & 8 & 83 & 33 & 142 & 50 & 383 & 133 \\
\hline
\end{tabular}

(1) Surface brightness limit used to determine the number of arcs; ${ }^{(2)}$ arc length-to-width ratio thresholds; ${ }^{(3)}$ redshift distribution and number count function, as introduced in Figs. 3 and 6.

brightness. The results are shown in Fig. 11 only for arcs with $d \geq d_{0}=10$.

\subsection{Submm wavelengths}

Given the poor resolution of current submm instruments, observational studies of submm arcs have not been possible so far, with only one submm arc candidate reported until now. This arc is supposed to be the brightest region of the extended submm source SMM J04542-0301, located in the core of the cluster MS0451.6-0305 (Borys et al. 2004; Berciano Alba et al. 2007; Berciano Alba et al. 2009, in press). However, higher resolution submm observations are required in order to confirm this hypothesis, and meaningful estimates of the length and width of this source, unfortunately, cannot yet be done. On the theoretical side, gravitational lensing of SMGs due to galaxy clusters was first studied by Blain (1997), using a circularly symmetric model of the cluster A2218 and the submm counts predicted by different galaxy evolution models. More recent studies (Cooray 1999; Paciga et al. 2009) also have been focused on predicting the number of submm lensed sources, but predictions for the abundance of submm arcs have never been attempted before.

For instance, in the work of Paciga et al. (2009), the authors employ the strong lensing analysis of the Millennium Simulation performed by Hilbert et al. (2007) (see also Hilbert et al. 2008) in order to compute (i) the average magnification of SMGs as a function of flux density; and (ii) the contribution to the differential number counts given by sources with different redshifts and magnifications. Hence, their results cannot be compared with ours in a straightforward way.

The only work with which we could try a tentative comparison is the one by Cooray (1999, CO99 hereafter), where the author provides number counts of (among others) gravitationally lensed submm sources as a function of their magnification. The clusters were modeled as Singular Isothermal Sphere (SIS henceforth) density profiles, which means that the image magnification equals its length-to-width ratio, as long as sources are circular and point-like. Since the sources that we are using are not circular, nor point-like, the following comparison should be taken with caution. The background submm sources were described by means of the redshift and number distributions observed in the Hubble Deep Field (HDF). Using a $\Lambda$ CDM cosmology, and considering cluster lenses with $M \geq 8.8 \times 10^{14} M_{\odot} h^{-1}$, CO99 predicted $\sim 500$ submm sources in the whole sky with lensing magnification larger than 10 and $S_{850 \mu \mathrm{m}} \geq 2 \mathrm{mJy}$.

On the other hand, our results indicate that, if no mass selection is applied and for $S_{850 \mu \mathrm{m}} \geq 2 \mathrm{mJy}$, we should find a few thousand arcs with $d \geq d_{0}=10$ in the whole sky. Restricting the cluster mass range to $M \geq 5 \times 10^{14} M_{\odot} h^{-1}$ reduces the number of arcs to $\sim 100$ at most. Matching the mass range of CO99 would only reduce the number of predicted arcs even further, hence being discrepant with CO99 predictions. Assuming that the source population we are considering is the same, we ascribe at least part of this disagreement to the fact that CO99 considers a very high normalization of the power spectrum $\left(\sigma_{8} \simeq 1.2\right)$, which was based on old studies of the cluster temperature function (Viana \& Liddle 1996). As shown in Fedeli et al. (2008), this results in greater strong lensing optical depth, and therefore to an over-prediction of the number of lensed images.

\subsection{Radio wavelengths}

The first observational search for radio arcs in galaxy clusters dates back to Bagchi \& Kapahi (1995), where the authors 


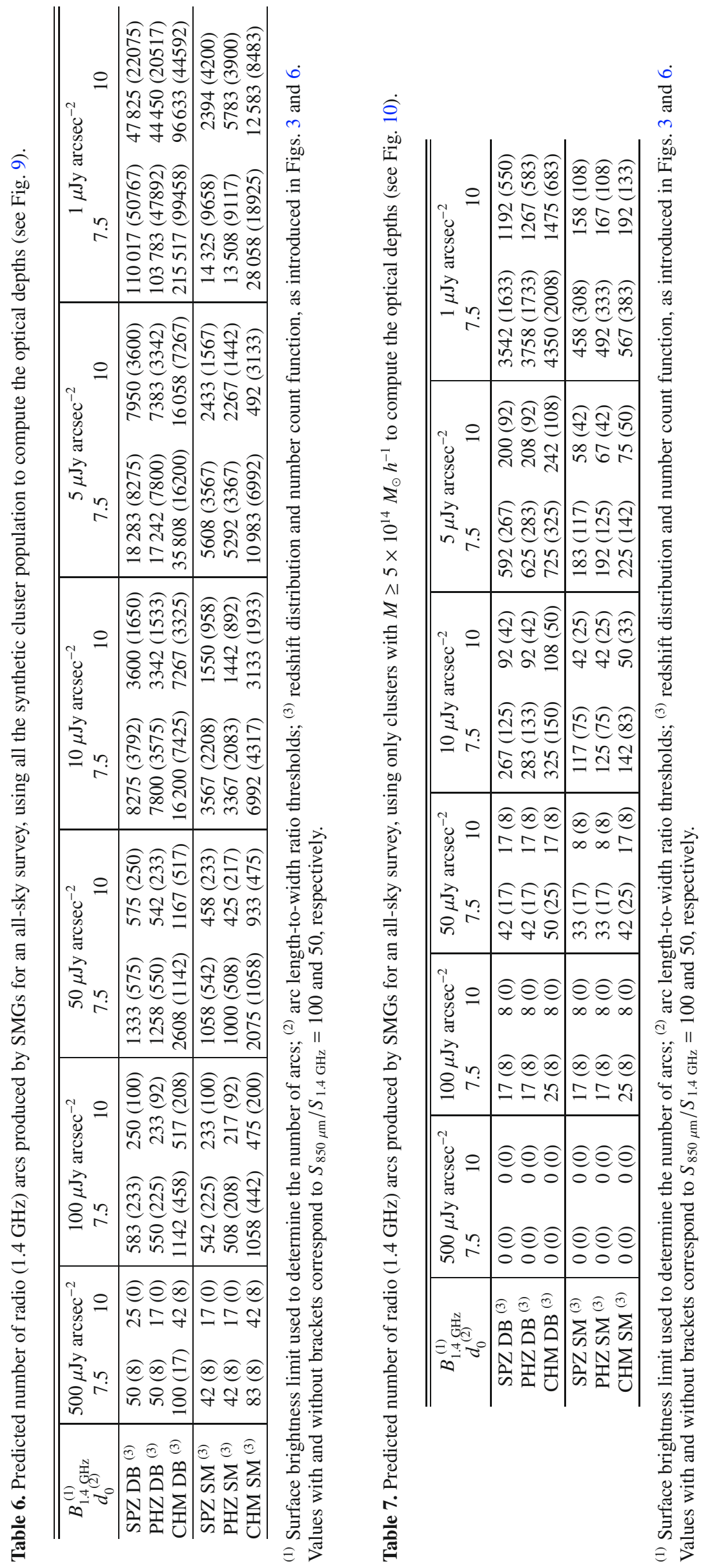



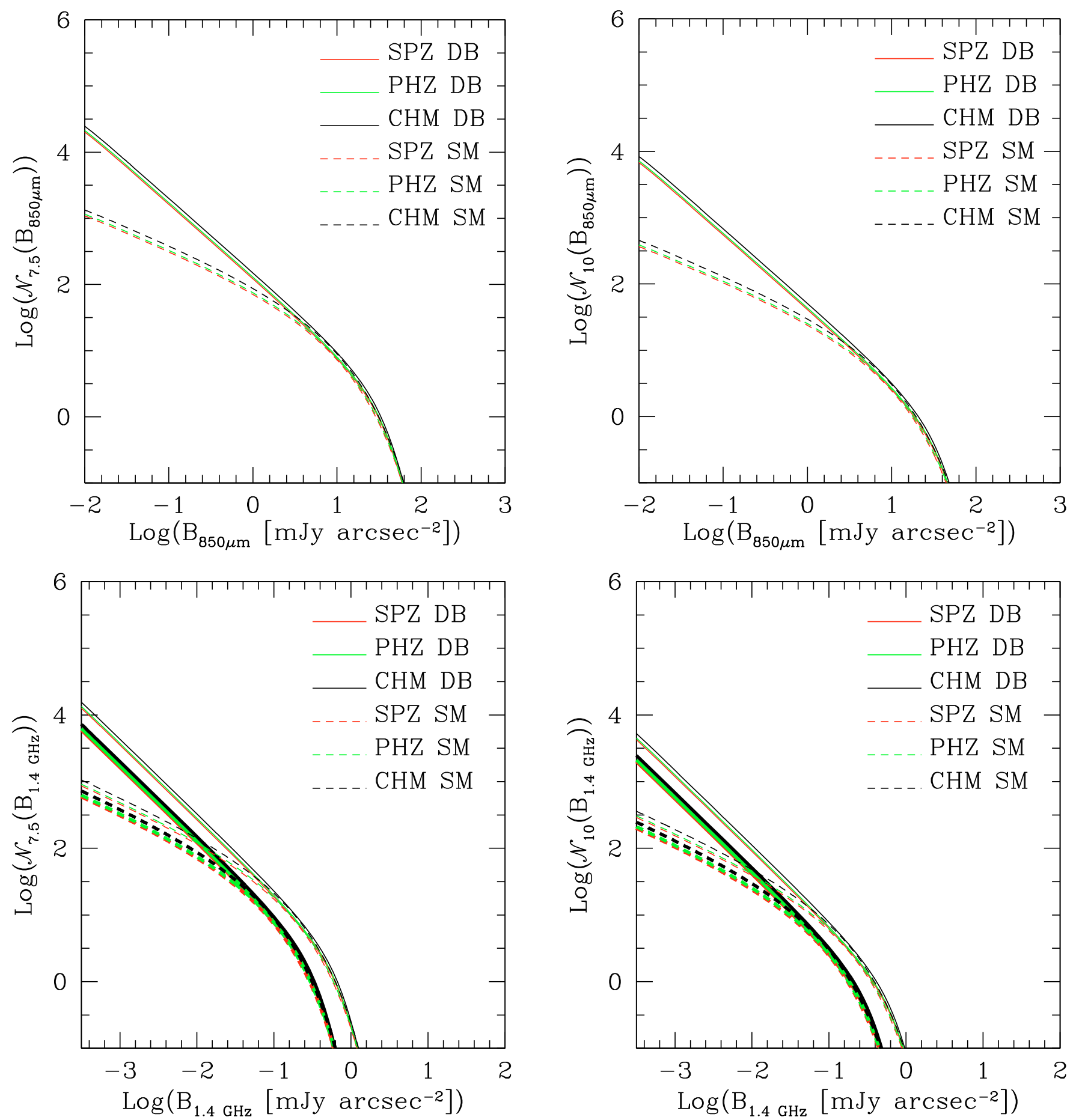

Fig. 10. The total number of arcs with $d \geq d_{0}=7.5$ (left panels) and $d \geq d_{0}=10$ (right panels) that are predicted to be observed in the whole sky above the surface brightness reported on the abscissa. Only clusters with mass $M \geq 5 \times 10^{14} M_{\odot} h^{-1}$ are included in the calculations. Results for each of the three input source redshift distributions and both source number counts adopted in this work are shown, according to the labels. Top panels refer to submillimeter number counts, while bottom panels refer to radio number counts (notice the different scale on the horizontal axis). The difference between thin and thick lines is as in Fig. 9.

considered a cluster sample of 46 objects with $z \lesssim 0.3$ observed, among others, at a wavelength of $20 \mathrm{~cm}$ with a sensitivity of $\sim 1 \mathrm{mJy}$. They claimed a systematic tangential alignment of radio images with respect to the cluster centers, and concluded that these are arclets or even giant arcs produced by background flat-spectrum radio sources. While their resolution was too poor to reliably measure arc morphological properties, these findings were subsequently questioned by Andernach et al. (1998), who performed a similar analysis on the Abell et al. (1989) cluster sample (ACO henceforth) with sources taken from the FIRST (Faint Images of the Radio Sky at Twenty-centimeters) catalogue (White et al. 1997), finding no evidence for a preferential alignment of radio images in the core of massive galaxy clusters. They also report a rough estimate of the abundance of strong lensing events that should be seen in ACO clusters, finding that for a statistically significant detection, the limiting flux density should 
be lowered to $\lesssim 0.1 \mathrm{mJy}$, in agreement with previous, more detailed estimates (Wu \& Hammer 1993). More recently, Phillips et al. (2001) searched for strong lensing events with large angular separations in the FIRST catalog, but none of their candidates turned out to be a real gravitational lens.

About $40 \%$ of the clusters included in the sample of Bagchi \& Kapahi (1995) have velocity dispersion $\sim 1000 \mathrm{~km} \mathrm{~s}^{-1}$. Assuming that cluster galaxies have the same velocity dispersion of the dark-matter particles (Gao et al. 2004; Biviano et al. 2006; Faltenbacher \& Diemand 2006, see however Colín et al. 2000), and adopting the simulation-calibrated scaling relation of Evrard et al. (2008), this corresponds to a mass of at least $\sim 8 \times$ $10^{14} M_{\odot} h^{-1}$. The rest of their clusters should have a mass $\gtrsim 4 \times$ $10^{14} M_{\odot} h^{-1}$. Assuming a limiting flux of $\sim 1 \mathrm{mJy}$ at $1.4 \mathrm{GHz}$, our predictions in Fig. 11 give $\$ 100$ giant radio arcs in the whole sky when the entire cluster population is considered, and only $\lesssim 1$ inside clusters with mass $M \geq 5 \times 10^{14} M_{\odot} h^{-1}$. Therefore, we find it quite unlikely that the detection claimed by Bagchi \& Kapahi (1995) is due to the radio emission from SMGs.

The previous considerations suggest the need to go to flux densities fainter than $1 \mathrm{mJy}$ in order to detect large radio arcs in galaxy clusters. This kind of implication is also supported by the results of Cooray (1998), which showed that the radio emission corresponding to large optical arcs in three out of the four cases he studied is $\$ 0.5 \mathrm{mJy}$. In the only optical arc with a secure radio counterpart in that work, namely arc $A 0$ in cluster $A 370$, the radio counterpart does not seem to be arc-shaped, which may be due to the radio emitting region being offset with respect with the optical emission or to resolution issues.

On the theoretical side, the statistics of radio arcs was first investigated by Wu \& Hammer (1993, WU93 hereafter). Their predictions were made using the evolutionary model of Dunlop \& Peacock (1990) to describe the radio luminosity function (dominated by starforming galaxies and AGNs preferentially located at $z \lesssim 1$ ) at $2.7 \mathrm{GHz}$ assuming a cosmological model with $\Omega_{\mathrm{m}, 0}=1$. Conversely, we focus on the radio counterparts of SMGs (preferentially located at $z \sim 2$ ) at $1.4 \mathrm{GHz}$ in a more modern $\Lambda$ CDM cosmology. As pointed out in Bartelmann et al. (1998), the optical depth for optical arcs produced by sources at $z_{\mathrm{s}} \sim 1$ grows by about one order of magnitude in going from an Einstein-de Sitter universe to a $\Lambda \mathrm{CDM}$ one. Therefore, we can use this prescription for comparison between both predictions. Note that, since the SED of SMGs is rather flat at radio wavelengths (spectral index $\sim 0.7$, Condon 1992), we expect only a small change in the flux density between $2.7 \mathrm{GHz}$ and $1.4 \mathrm{GHz}$.

In their calculations, WU93 only considered clusters with $z \lesssim 0.6$ and a galaxy velocity dispersion $>800 \mathrm{~km} \mathrm{~s}^{-1}$, which corresponds to a mass $\gtrsim 3.5 \times 10^{14} M_{\odot} h^{-1}$. After applying the cosmology correction mentioned before, their predicted number of giant radio arcs for the whole sky is (i) $\sim 1000$ if $S_{2.7 \mathrm{GHz}} \gtrsim$ $10 \mu \mathrm{Jy}$; (ii) $\lesssim 200$ if $S_{2.7 \mathrm{GHz}}>0.1 \mathrm{mJy}$ and (iii) very small if $S_{2.7 \mathrm{GHz}}>$ a few mJy (such that none should be detected in surveys in the literature at that time). Considering only clusters with mass $\geq 5 \times 10^{14} M_{\odot} h^{-1}$ (which have a redshift range comparable to the one used in WU93), our predicted number of radio arcs with $d \geq d_{0}=10$ produced by SMGs in the whole sky is (i) $\sim 100-1000$ if $S_{1.4 \mathrm{GHz}}>10 \mu \mathrm{Jy}$; (ii) few tens if $S_{1.4 \mathrm{GHz}}>0.1 \mathrm{mJy}$ and (iii) very small if $S_{1.4 \mathrm{GHz}}>$ a few mJy.

Our results may thus seem compatible with those of WU99, considering that the number density of SMGs is certainly smaller than the number density of the entire radio source population. This inference is however not conclusive, since WU99 are using sources at rather low-redshift. We cannot say whether considering their same redshift distribution and number counts would lead to a discrepant result.

In CO99 there is also a study about strongly magnified radio sources, analogous to the submm sources. It is found that $\sim 20$ sources with amplification larger than 10 should be found with $S_{1.4 \mathrm{GHz}} \geq 10 \mu \mathrm{Jy}$. For the same parameters, we find in our high-mass cluster study a number ranging from $\sim 100$ up to $\sim 1000$, which is much larger than the findings of CO99. This discrepancy should be even more enhanced if the two calculations are reduced to the same $\sigma_{8}$. The origin of this discrepancy is not clear, although it might be related to the higher mass threshold that they adopt, and the fact that the redshift distribution considered by CO99 peaks at $z \sim 1$ instead of at $z \sim 2$. It should also be noted that CO99 attribute their finding many fewer radio arcs than WU93 to the different number count evolution adopted.

Our calculations of the abundance of radio and submm arcs are more accurate than the works discussed above for different reasons, mainly the different modeling of the cluster population. To start with, in the literature, investigators often consider all lenses as isolated, spherically symmetric density distributions. Wu \& Hammer (1993) investigate the effect of elliptical mass distributions, but only on the magnification pattern and not on the efficiency for the production of large arcs. On the other hand, we included the effect of asymmetries, substructures and cluster mergers, that all have been found to be important to augment arc statistics. Next, we used an NFW density profile to model individual lenses, which is a good representation of average darkmatter dominated objects like galaxy clusters, while other works have often considered SIS or SIS-like profiles, which are more suitable for galaxy lensing. While for a SIS lens model the image magnification equals the length-to-width ratio, it is known to produce fewer gravitational arcs with respect to the more realistic NFW profile (Meneghetti et al. 2003).

\section{Summary and conclusions}

The advent of the high resolution submm facilities ALMA and CCAT, and the major technological development that radio interferometry is currently undergoing (e.g., $e$-MERLIN, EVLA and SKA) will make possible the study of radio and submm giant arcs produced by clusters of galaxies. In particular, the study of giant arcs produced by submm galaxies (SMGs) seems particularly promising for at least two reasons.

- It provides the opportunity to detect and spatially resolve the morphologies and internal dynamics of this population of dust obscured high-redshift star-forming galaxies, which is very difficult to study in the optical.

- It can provide information about the formation and evolution of the high redshift cluster population, by means of arc statistics studies.

To assess the prospects for these kind of studies, we provided theoretical predictions on the abundance of gravitational arcs produced by the SMG population at radio $(1.4 \mathrm{GHz})$ and submm $(850 \mu \mathrm{m})$ wavelengths, greatly improving the accuracy of the results with respect to the first few studies carried out a decade (or more) ago. The advantage of radio observations is that the angular resolution and sensitivity provided by interferometers like $e$-MERLIN and EVLA are already (or will very soon be) at the level required for these kind of studies. However, these frequencies do not benefit from the same favorable $K$-correction as $\mathrm{mm} / \mathrm{submm}$ wavelengths do, which will make the latter a more interesting tool for studying high-redshift clusters as soon as the 

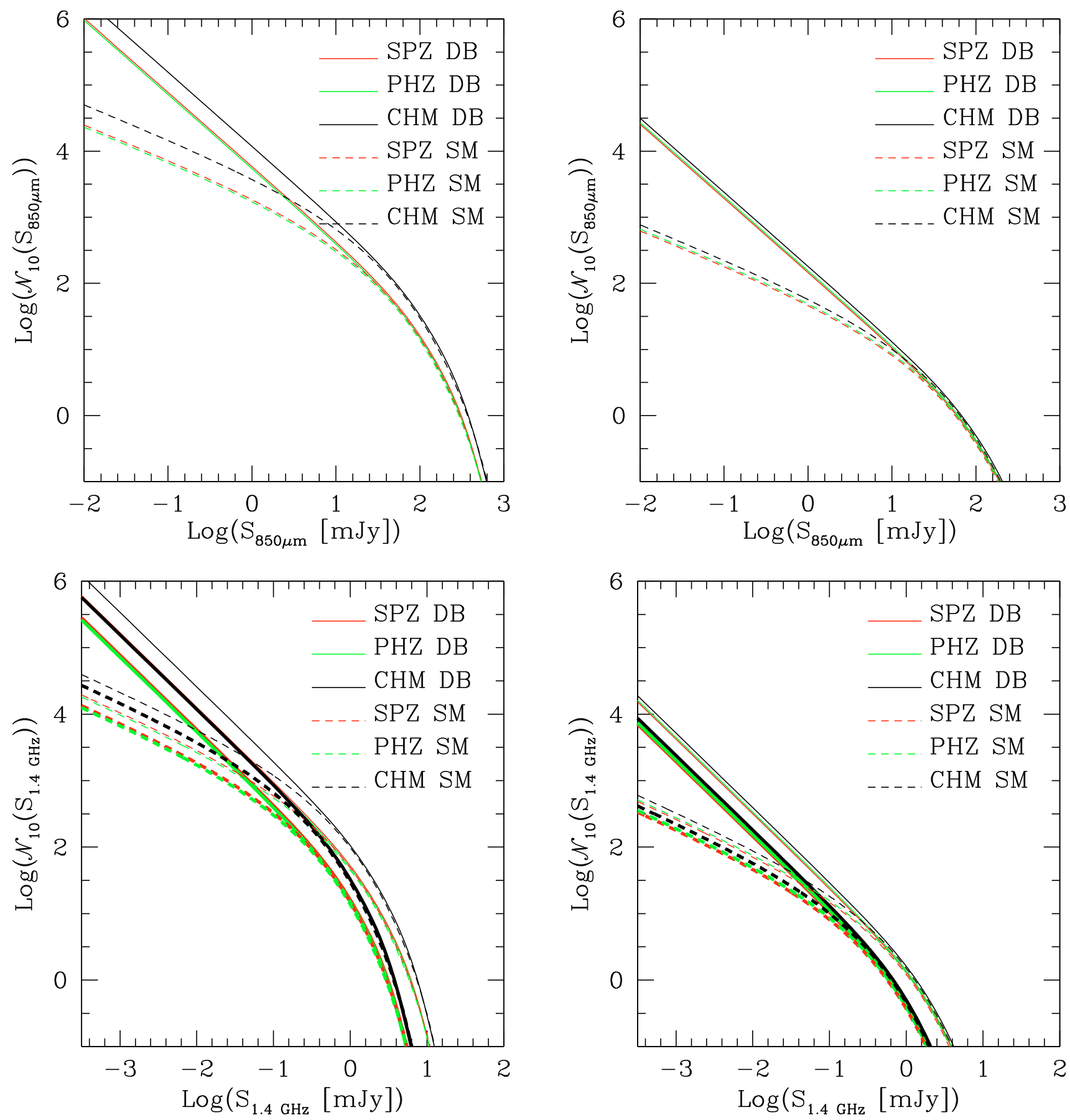

Fig. 11. The number of arcs with $d \geq d_{0}=10$ predicted to be observed in the whole sky as a function of the submm flux density at $850 \mu \mathrm{m}$ (top two panels) and radio flux density at $1.4 \mathrm{GHz}$ (bottom two). The left panels refer to all clusters in the sample, while the right ones shows results when only clusters with mass $M \geq 10^{14} M_{\odot} h^{-1}$ are considered. Different line styles and colors refer to different number count models and source redshift distributions. In the two bottom panels, the thin lines assume $S_{1.4 \mathrm{GHz}}=S_{850 \mu \mathrm{m}} / 50$, while the thick lines refer to $S_{1.4 \mathrm{GHz}}=S_{850 \mu \mathrm{m}} / 100$.

resolution of sub-mm observations is good enough. The calculation of the number of arcs produced by a background source population requires four main ingredients: (i) the source shape and size; (ii) the source redshift distribution; (iii) the cumulative source number counts; and (iv) a model of the cluster population.

The model of the cluster population used in this work was based on the extended Press \& Schechter (1974) formalism and made use of an NFW dentity profile to describe each cluster lens. It also included the effect of asymmetries, substructures and cluster mergers, which have been found to play an important role in arc lensing statistics.
Based on current radio/CO observations and the FIR/radio correlation, we have characterized the typical size of the radio and submm emitting regions of SMGs with an effective radius $R_{\mathrm{e}}=0.25^{\prime \prime}$, and an axis ratio that varies within the interval $b / a \in$ $[0.3,1]$. Resolving all the arcs produced by this kind of sources will require $\sim 0.2^{\prime \prime}$ resolution.

Since the most accurate redshift distribution of SMGs available (Chapman et al. 2005) is based on observations of the radio detected members (which is biased against $z \geq 3$ sources), we used three different functions to quantify the effect of a high redshift tail on the predicted number of arcs. The results indicate 
that this effect is less than a factor two if we consider all simulated clusters during the calculations, and negligible if we only consider massive clusters.

The submm source number counts used in this work correspond to the joint fit of the (bright) SHADES survey counts and the (faint) Leiden SCUBA Lens Survey counts presented in Knudsen et al. (2008). To account for the uncertainty in the low flux end, predictions were made for the best fit to the data, and the shallowest fit consistent with the data. Note that, although only $\sim 60 \%$ of the observed SMGs have being detected in radio, the next generation of radio interferometers will be able to detect the radio counterparts of all SMGs with $S_{850 \mu} \geq 5 \mathrm{mJy}$. Therefore, the radio number counts of SMGs have been derived by scaling the submm counts using representative upper and lower limits of the $S_{850 \mu \mathrm{m}} / S_{1.4 \mathrm{GHz}}$ ratio for SMGs at $z \sim 2$ taken from Chapman et al. (2005).

Our calculations show that a submm all-sky survey with a sensitivity of $1 \mathrm{mJy} \operatorname{arcsec}^{-2}$ will detect hundreds of arcs with a $5 \sigma$ significance. In the radio, this number can be achieved with a sensitivity of $10-20 \mu \mathrm{Jy} \operatorname{arcsec}^{-2}$. Obtaining a statistically significant sample of thousands of arcs would require sensitivities of $0.1 \mathrm{mJy} \operatorname{arcsec}^{-2}$ in the submm and $1 \mu \mathrm{Jy} \operatorname{arcsec}^{-2}$ in the radio. However, if only massive clusters $\left(M \geq 5 \times 10^{14} M_{\odot} h^{-1}\right)$ are considered in the calculations, the predicted number of arcs is reduced by about an order of magnitude.

Besides the many uncertainties involved in the theoretical predictions presented here, the main challenge in designing a future survey for radio/submm arc statistics studies will be in finding the best compromise between survey area, depth and resolution, three issues that affect the arc detectability in different manners. We believe that this work provides a significant step forward in this direction.

Acknowledgements. We are grateful to M. Bartelmann, A. Blain and L. V. E. Koopmans for reading the manuscript and for many useful comments. We also would like to thank M. Swinbank, for providing us the histograms presented in Fig. 4, their Gaussian fits, and the predictions from his evolutionary model, and $\mathrm{K}$. K. Kundsen for providing us with the observational data presented in Fig. 5. We also acknowledge stimulating conversation with $\mathrm{M}$. Bonamente, M. Brentjens, M. Joy, A. F. Loenen and I. Prandoni. We wish to thank the anonymous referee for useful remarks that allowed us to improve the presentation of our work. C.F. acknowledges financial contributions from contracts ASI-INAF I/023/05/0 and ASI-INAF I/088/06/0.

\section{References}

Abell, G. O., Corwin, Jr., H. G., \& Olowin, R. P. 1989, ApJS, 70, 1

Alexander, D. M., Bauer, F. E., Brandt, W. N., et al. 2003, AJ, 125, 383

Alexander, D. M., Bauer, F. E., Chapman, S. C., et al. 2005, ApJ, 632, 736

Andernach, H., Gubanov, A. G., \& Slee, O. B. 1998, in Observational Cosmology with the New Radio Surveys, ed. M. N. Bremer, N. Jackson, \& I. Perez-Fournon, Astrophys. Space Sci. Library, 226, 107

Aretxaga, I., Hughes, D. H., Coppin, K., et al. 2007, MNRAS, 379, 1571 (AR07)

Austermann, J. E., Dunlop, J. S., Perera, T. A., et al. 2009, MNRAS, accepted [arXiv:0907.1093]

Bagchi, J., \& Kapahi, V. K. 1995, J. Astrophys. Astron. Suppl., 16, 131

Barger, A. J., Cowie, L. L., Sanders, D. B., et al. 1998, Nature, 394, 248

Bartelmann, M. 1996, A\&A, 313, 697

Bartelmann, M., \& Weiss, A. 1994, A\&A, 287, 1

Bartelmann, M., \& Schneider, P. 2001, Phys. Rep., 340, 291

Bartelmann, M., Huss, A., Colberg, J. M., Jenkins, A., \& Pearce, F. R. 1998, A\&A, 330, 1

Bartelmann, M., Meneghetti, M., Perrotta, F., Baccigalupi, C., \& Moscardini, L. 2003, A\&A, 409, 449

Berciano Alba, A., Garrett, M. A., Koopmans, L. V. E., \& Wucknitz, O. 2007, A\&A, 462, 903
Biggs, A. D., \& Ivison, R. J. 2008, MNRAS, 385, 893 (BI08) Biviano, A., Murante, G., Borgani, S., et al. 2006, A\&A, 456, 23 Blain, A. W. 1996, MNRAS, 283, 1340

Blain, A. W. 1997, MNRAS, 290, 553

Blain, A. W., \& Longair, M. S. 1993, MNRAS, 264, 509

Blain, A. W., \& Longair, M. S. 1996, MNRAS, 279, 847

Blain, A. W., Smail, I., Ivison, R. J., Kneib, J.-P., \& Frayer, D. T. 2002, Phys. Rep., 369, 111

Bond, J. R., Cole, S., Efstathiou, G., \& Kaiser, N. 1991, ApJ, 379, 440

Borys, C., Chapman, S., Halpern, M., \& Scott, D. 2003, MNRAS, 344, 385

Borys, C., Chapman, S., Donahue, M., et al. 2004, MNRAS, 352, 759 (B04)

Chapman, S. C., Scott, D., Borys, C., \& Fahlman, G. G. 2002, MNRAS, 330, 92

Chapman, S. C., Helou, G., Lewis, G. F., \& Dale, D. A. 2003, ApJ, 588, 186

Chapman, S. C., Smail, I., Windhorst, R., Muxlow, T., \& Ivison, R. J. 2004, ApJ, 611,732

Chapman, S. C., Blain, A. W., Smail, I., \& Ivison, R. J. 2005, ApJ, 622, 772 (CH05)

Colín, P., Klypin, A. A., \& Kravtsov, A. V. 2000, ApJ, 539, 561

Condon, J. J. 1992, ARA\&A, 30, 575

Cooray, A. R. 1998 [arXiv:astro-ph/9802108]

Cooray, A. R. 1999, A\&A, 341, 653 (CO99)

Coppin, K., Chapin, E. L., Mortier, A. M. J., et al. 2006, MNRAS, 372, 1621

Cowie, L. L., Barger, A. J., \& Kneib, J.-P. 2002, AJ, 123, 2197

Dolag, K., Bartelmann, M., Perrotta, F., et al. 2004, A\&A, 416, 853

Dubuisson, M. P., \& Jain, A. K. 1994, Proceedings of the 12th IAPR International Conference on Pattern Recognition, 1, 566

Dunkley, J., Komatsu, E., Nolta, M. R., et al. 2009, ApJS, 180, 306

Dunlop, J. S., \& Peacock, J. A. 1990, MNRAS, 247, 19

Eales, S., Lilly, S., Gear, W., et al. 1999, ApJ, 515, 518

Evrard, A. E., Bialek, J., Busha, M., et al. 2008, ApJ, 672, 122

Faltenbacher, A., \& Diemand, J. 2006, MNRAS, 369, 1698

Fedeli, C., \& Bartelmann, M. 2007, A\&A, 461, 49

Fedeli, C., Meneghetti, M., Bartelmann, M., Dolag, K., \& Moscardini, L. 2006, A\&A, 447, 419

Fedeli, C., Bartelmann, M., Meneghetti, M., \& Moscardini, L. 2008, A\&A, 486, 35

Fixsen, D. J., Dwek, E., Mather, J. C., Bennett, C. L., \& Shafer, R. A. 1998, ApJ, 508,123

Gao, L., De Lucia, G., White, S. D. M., \& Jenkins, A. 2004, MNRAS, 352, L1

Garrett, M. A. 2002, A\&A, 384, L19

Genzel, R., Baker, A. J., Tacconi, L. J., et al. 2003, ApJ, 584, 633

Gladders, M. D., Hoekstra, H., Yee, H. K. C., Hall, P. B., \& Barrientos, L. F. 2003, ApJ, 593, 48

Gottlöber, S., \& Yepes, G. 2007, ApJ, 664, 117

Greve, T. R., Bertoldi, F., Smail, I., et al. 2005, MNRAS, 359, 1165

Harwit, M., \& Pacini, F. 1975, ApJ, 200, L127

Helou, G., Soifer, B. T., \& Rowan-Robinson, M. 1985, ApJ, 298, L7

Hilbert, S., White, S. D. M., Hartlap, J., \& Schneider, P. 2007, MNRAS, 382, 121

Hilbert, S., White, S. D. M., Hartlap, J., \& Schneider, P. 2008, MNRAS, 386, 1845

Holland, W. S., Robson, E. I., Gear, W. K., et al. 1999, MNRAS, 303, 659

Hughes, D. H., Serjeant, S., Dunlop, J., et al. 1998, Nature, 394, 241

Ibar, E., Cirasuolo, M., Ivison, R., et al. 2008, MNRAS, 386, 953

Ivison, R. J., Greve, T. R., Smail, I., et al. 2002, MNRAS, 337, 1

Keeton, C. R. 2001, ApJ, 562, 160

Kneib, J.-P., van der Werf, P. P., Kraiberg Knudsen, K., et al. 2004, MNRAS, 349,1211

Knudsen, K. K., van der Werf, P. P., \& Kneib, J.-P. 2008, MNRAS, 384, 1611 (KN08)

Komatsu, E., Dunkley, J., Nolta, M. R., et al. 2009, ApJS, 180, 330

Kovács, A., Chapman, S. C., Dowell, C. D., et al. 2006, ApJ, 650, 592

Lacey, C., \& Cole, S. 1993, MNRAS, 262, 627

Lewis, G. F., Chapman, S. C., \& Helou, G. 2005, ApJ, 621, 32

Li, G.-L., Mao, S., Jing, Y. P., et al. 2005, ApJ, 635, 795

Lilly, S. J., Eales, S. A., Gear, W. K. P., et al. 1999, ApJ, 518, 641

Luppino, G. A., Gioia, I. M., Hammer, F., Le Fèvre, O., \& Annis, J. A. 1999, A\&AS, 136, 117

Meneghetti, M., Bolzonella, M., Bartelmann, M., Moscardini, L., \& Tormen, G. 2000, MNRAS, 314, 338

Meneghetti, M., Bartelmann, M., \& Moscardini, L. 2003, MNRAS, 340, 105

Meneghetti, M., Bartelmann, M., Dolag, K., et al. 2005, A\&A, 442, 413

Michałowski, M. J., Hjorth, J., \& Watson, D. 2009, MNRAS, submitted [arXiv: 0905 . 4499]

Miralda-Escude, J. 1993, ApJ, 403, 497

Mortier, A. M. J., Serjeant, S., Dunlop, J. S., et al. 2005, MNRAS, 363, 563

Muxlow, T. W. B., Richards, A. M. S., Garrington, S. T., et al. 2005, MNRAS, 358,1159 
Navarro, J. F., Frenk, C. S., \& White, S. D. M. 1995, MNRAS, 275, 56 Navarro, J. F., Frenk, C. S., \& White, S. D. M. 1996, ApJ, 462, 563 Navarro, J. F., Frenk, C. S., \& White, S. D. M. 1997, ApJ, 490, 493 Oguri, M., Hennawi, J. F., Gladders, M. D., et al. 2009, ApJ, 699, 1038 Paciga, G., Scott, D., \& Chapin, E. L. 2009, MNRAS, 395, 1153 Phillips, P. M., Browne, I. W. A., \& Wilkinson, P. N. 2001, MNRAS, 321, 187 Press, W. H., \& Schechter, P. 1974, ApJ, 187, 425

Puget, J.-L., Abergel, A., Bernard, J.-P., et al. 1996, A\&A, 308, L5

Rzepecki, J., Lombardi, M., Rosati, P., Bignamini, A., \& Tozzi, P. 2007, A\&A, 471,743

Schechter, P. 1976, ApJ, 203, 297

Schmidt, R. W., \& Allen, S. W. 2007, MNRAS, 379, 209

Smail, I., Hogg, D. W., Yan, L., \& Cohen, J. G. 1995, ApJ, 449, L105

Smail, I., Ivison, R. J., \& Blain, A. W. 1997, ApJ, 490, L5

Smail, I., Ivison, R. J., Blain, A. W., \& Kneib, J.-P. 2002, MNRAS, 331, 495

Smail, I., Chapman, S. C., Blain, A. W., \& Ivison, R. J. 2004, ApJ, 616, 71

Somerville, R. S., \& Kolatt, T. S. 1999, MNRAS, 305, 1
Swinbank, A. M., Chapman, S. C., Smail, I, et al. 2006, MNRAS, 371, 465 Swinbank, A. M., Bower, R. G., Smith, G. P., et al. 2007, MNRAS, 376, 479 Swinbank, A. M., Lacey, C. G., Smail, I., et al. 2008, MNRAS, 391, 420 (SW08) Tacconi, L. J., Neri, R., Chapman, S. C., et al. 2006, ApJ, 640, 228

Torri, E., Meneghetti, M., Bartelmann, M., et al. 2004, MNRAS, 349, 476 van der Kruit, P. C. 1973, A\&A, 29, 263

van Kampen, E., Percival, W. J., Crawford, M., et al. 2005, MNRAS, 359, 469 Viana, P. T. P., \& Liddle, A. R. 1996, MNRAS, 281, 323

Vlahakis, C., Eales, S., \& Dunne, L. 2007, MNRAS, 379, 1042

Wambsganss, J., Cen, R., \& Ostriker, J. P. 1998, ApJ, 494, 29 Wambsganss, J., Bode, P., \& Ostriker, J. P. 2005, ApJ, 635, L1

Webb, T. M., Eales, S. A., Lilly, S. J., et al. 2003, ApJ, 587, 41

White, R. L., Becker, R. H., Helfand, D. J., \& Gregg, M. D. 1997, ApJ, 475, 479 Wu, X.-P., \& Hammer, F. 1993, MNRAS, 262, 187 (WU93)

Younger, J. D., Fazio, G. G., Wilner, D. J., et al. 2008, ApJ, 688, 59 Zaritsky, D., \& Gonzalez, A. H. 2003, ApJ, 584, 691 